\title{
Longitudinal drift of substorm electrons as the reason of impulsive precipitation events and VLF emissions
}

\author{
A. A. Lubchich ${ }^{1}$, A. G. Yahnin ${ }^{1}$, E. E. Titova ${ }^{1}$, A. G. Demekhov ${ }^{2}$, V. Yu. Trakhtengerts ${ }^{2}$, J. Manninen ${ }^{3}$, and \\ T. Turunen ${ }^{3}$ \\ ${ }^{1}$ Polar Geophysical Institute, Apatity, Russia \\ ${ }^{2}$ Institute of Applied Physics, Nizhny Novgorod, Russia \\ ${ }^{3}$ Geophysical Observatory, FIN-99600 Sodankylä, Finland
}

Received: 22 March 2006 - Revised: 30 June 2006 - Accepted: 24 August 2006 - Published: 20 October 2006

\begin{abstract}
Using the data from satellite CRRES and three geostationary LANL spacecraft, the propagation of an electron cloud from midnight to the evening sector is investigated. An electron cloud was injected during a weak isolated substorm that developed on a quiet geomagnetic background. It is found that within the local time sector from 03:00 until at least 08:00 MLT, the propagation of electrons at perpendicular pitch-angles is well described by a simple model of drift in the dipole magnetic field. The flux levels in the field-aligned electrons increase simultaneously with the flux at perpendicular pitch angles, which is attributed to the pitch angle diffusion by the whistler mode. This pitch-angle diffusion leads to precipitation of electrons from a drifting cloud and an increase in the ionospheric electron density, simultaneously observed above Troms $\varnothing$, Norway, by the EISCAT UHF radar in the morning sector (04:40-05:25 MLT). The precipitation develops as quasi-periodic pulses with a period of about $100 \mathrm{~s}$. We discuss the models of pulsating precipitation due to the whistler cyclotron instability and show that our observations can be explained by such a model.
\end{abstract}

Keywords. Magnetospheric physics (Energetic particles, precipitating; Storms and substorms) - Space Plasma Physics (Wave-particle interactions; Waves and instabilities)

\section{Introduction}

During a substorm expansion phase, the plasma sheet particles are accelerated and injected into the midnight sector of the Earth's inner magnetosphere, into the region of a quasidipole magnetic field. Particle injections are a fundamental signature of substorms. A sudden increase in the particle flux appearing simultaneously at different energies is called a "dispersionless injection", and the region in space where

Correspondence to: A. A. Lubchich

(lubchich@pgi.kolasc.net.ru) the dispersionless injection occurs is called the "injection region". Properties of substorm injections have been investigated by many authors. The depth of the injection boundary, i.e. the radial distance of the closest approach of accelerated particles to the Earth, varies within a wide range (from $\sim 4 R_{E}$ up to $\sim 10 R_{E}$ ). In particular, Lopez et al. (1990), using satellite AMPTE/CCE data $(5.5<L<9.5)$, and Reeves et al. (1996), using the data from the CRRES mission (4 $<L<7$ ), investigated the probability of injection observation depending on the L-shell. They established that the probability is maximum at $L=8$ and almost monotonicaly decreases approaching the Earth. Calculating the delay between the injection seen by the LANL geostationary satellite and by CRRES when the two spacecraft were within $\pm 1 \mathrm{~h}$ of local time, Reeves et al. (1996) have shown that the injection region propagates earthward with the velocity $\sim 24 \mathrm{~km} / \mathrm{s}$ (i.e., to move by $1 R_{E}$ it takes approx. $4.5 \mathrm{~min}$ ). The dispersionless injections, both in energy and pitch-angle, can be observed at the nightside within a wide local time sector from 18:00 to 06:00 LT. The dispersionless injection occurrence has been statistically found to be centered in the pre-midnight sector (Friedel et al., 1996; Birn et al., 1997). Both electrons and ions from about tens to hundreds of $\mathrm{keV}$ are injected. The region of injection can be rather extended in longitude (by $60^{\circ}$ and more). Birn et al. (1997), using the superposed epoch method, studied delays between ion and electron dispersionless injections depending on the longitudinal sector of the injection region. Five different categories of events have been distinguished. Moving from the evening side to the morning side, one would observe in the following order: 1) ion injection without electron injection; 2) ion injection followed by electron injection with a few minutes delay; 3 ) simultaneous ion and electron injections; 4) electron injection followed by a proton injection with a few minutes delay; 5) electron injection without ion injection.

The magnetic field inhomogeneity in the inner magnetosphere gives rise to the separation of injected electrons and

Published by Copernicus GmbH on behalf of the European Geosciences Union. 
ions. Because of the radial gradient and curvature of magnetic field lines, the high energy ions drift westward and the high energy electrons drift eastward. The magnetic drift velocity is directly proportional to the particle energy. Therefore, the energy dispersion in a drifting particle cloud increases with the distance from the midnight meridian. This is observed well by geostationary satellites spaced in longitude. In the dipole magnetic field the bounce-averaged velocity of magnetic drift, i.e. the sum of gradient and curvature drifts, monotonously increases with the pitch angle of a particle (e.g. Lyons and Williams, 1984). However, in reality, the motion pattern is often more complex. The expected dispersion on pitch angles in a drifting cloud is indeed observed near the midnight sector (e.g. Walker et al., 1978). But at a significant distance from the midnight meridian, particles with small pitch angles can be detected even before particles with greater pitch angles as noted by Torkar et al. (1987) for drifting electrons and by Walker et al. (1978) for drifting protons). Åsnes et al. (2005) investigated the statistical properties of the pitch-angle distribution of substorm electrons in a drifting cloud using particle measurements on board LANL geostationary satellites. It has been shown that an increase in the anisotropy some time after the first cloud arrival is common for all local times.

At early local times (02:00-04:00 MLT), the anisotropy reaches a maximum approximately in an hour after the beginning of the flux increase, and the average duration of the cloud of particles with an energy of $36-47 \mathrm{keV}$ is one hour and a half. At later MLT, the anisotropy maximum is reached later and has a smaller amplitude. Finally, at 10:0012:00 MLT, most events do not show a significant increase in anisotropy. Two explanations can be proposed for observable peculiarities of the pitch-angle distribution of drifting substorm particles. One is related to the differences in drift orbits for different pitch-angles and energies. The second process affecting the pitch angle distribution is the pitch-angle diffusion.

Many authors modelled the drift motion of particles in the magnetosphere. The drift trajectories have a simplest form in the dipole approximation of the magnetic field (e.g. Roederer, 1970; Lyons and Williams, 1984). In this model, the particle motion has a remarkable property, namely, a particle of any energy and with any pitch-angle outside of the loss cone will remain at the same L-shell during its longitudinal drift. Therefore, when analysing the longitudinal drift in the dipole magnetic field, one does not need additional hypotheses about the radial structure of the injection region.

Many authors investigated the particle motion in more realistic models of the magnetosphere. For example, Shukhtina and Sergeev (1991) analysed the particle motion in the model T-89 (Tsyganenko, 1989); Takahashi and Iyemori (1989) calculated the guiding centre motion of a particle in the magnetic field model of Mead and Fairfield (1975); Lyons and Williams (1984) gave the detailed calculations of drift trajectories in the dipole magnetic field, taking into account electric fields of convection and corotation. As is known, in the non-dipole magnetic field the drift shell splitting is observed (Roederer, 1970), i.e. during the longitudinal drift in the magnetospheric magnetic field the particle moves from one L-shell to another, and this motion depends on the particle pitch angle. Drifting from the nightside to the dayside, the particles with small pitch-angles move towards the Earth, and particles with greater pitch-angles, on the contrary, move away from the Earth. As calculations by Shukhtina and Sergeev (1991) show, at the moderate geomagnetic activity $\left(K_{p}=3\right)$ the divergence of $100-\mathrm{keV}$ particles with different pitch angles, starting from the midnight meridian on geostationary orbit, can reach $2-4 R_{E}$. Thus, the effect of the drift shell splitting is essential. Unlike the motion in the dipole field, the longitudinal drift velocity in more realistic magnetospheric field models is azimuthally non-uniform. In the midnight sector the drift velocity is higher than at noon. For "geostationary" particles with energies of $100 \mathrm{keV}$, the velocities can differ by a factor of 1.5-2 (Shukhtina and Sergeev, 1991). However, modelling of particle motion in the realistic magnetic field models could not explain the inverse pitchangle dispersion experimentally measured in some cases (see Walker et al., 1978 and Torkar et al., 1987).

As is known, the drift in the electric field does not depend on the charge, mass, pitch angle and energy of a particle, and depends only on the magnitude of the electric field and the mutual orientation of electric and magnetic fields. In the inner magnetosphere, the electric drift of charged particles has an eastward direction coincident with the direction of the magnetic drift of the electrons. Therefore, the electric field distorts proton drift trajectories stronger. The electric field of corotation, directed radially to the Earth, equals $\sim 0.33 \mathrm{mV} / \mathrm{m}$ at the geostationary orbit. The estimation of the electric field convection, directed from the morning side to the evening side, gives $\sim 0.25 \mathrm{mV} / \mathrm{m}$ (Lyons and Williams, 1984). In the morning sector of the magnetosphere, where these two fields are simply added, particles with energies $\sim 8 \mathrm{keV}$ at the geostationary orbit have identical velocities of magnetic and electric drifts. Therefore, for particles with energies of some tens of $\mathrm{keV}$ and below, it is necessary to consider the influence of the electric field, whereas for particles with greater energies, such influence can be neglected. Calculations by Lyons and Williams (1984) confirm that the influence of the convection and corotation electric field on drift trajectories of 30-keV electrons in the Earth's inner magnetosphere is insignificant. Roederer and Hones (1974) have shown that models with a stationary electric and magnetic field cannot lead to the generation of a drifting particle cloud. They have considered the model including the dipole magnetic field, the constant electric field of convection and corotation, and the variable electric field consisting of the homogeneous dawn-dusk field and the azimuthal field localised in the midnight sector. The variable electric field increased (within $10 \mathrm{~min}$ ) rapidly to the maximum value and slowly (within two hours) decreased to zero. By varying the 
parameters of the electric field model, it is possible to explain the experimentally observable form of the drifting cloud of the substorm injected particles. However, within the limits of such a model it is difficult to explain the complex character of the observed dispersion of the pitch-angle distribution. Birn et al. (1998), Li et al. (2003), and Sarris and Li (2005) simulated the injection of equatorial energetic particles (i.e. with the $90^{\circ}$ pitch-angle) into the inner magnetosphere, as a consequence of the near-Earth magnetic reconnection. The simulation allowed to reproduce major characteristics of the dispersionless injection, in particular, the existence of five different categories of observed injection events (see Birn et al., 1997). Simulating one substorm injection (a case study) Li et al. (2003), and Sarris and Li (2005) obtained equatorial energetic $(>100 \mathrm{keV})$ electron fluxes at different local time and reproduced the dispersion feature, drift echo, width and shape of the fluxes, observed by LANL satellites. However the electron in the simulation drift faster than the measurements.

The point of view, that the variation of anisotropy within the drifting cloud of substorm injected particles is the result of the pitch-angle diffusion because of the resonance interaction of energetic particles with VLF waves (e.g., Torkar et al., 1987; Åsnes et al., 2005; Lyons and Williams, 1984) seems to be more realistic. In particular, Åsnes et al. (2005) analyse statistical properties of substorm-injected electron clouds at geosynchronous orbit. They come to conclusion that from midnight till noon the pitch-angle diffusion by interaction with waves is the basic mechanism of formation of the electron pitch-angle distribution at energies greater than $10 \mathrm{keV}$, whereas after local noon the pitch-angle distributions evolve due to differential drift effects. Many experimental results evidence this point of view. As an example it is possible to refer to the properties of the aurora, being the typical phenomenon in the morning sector during a substorm recovery phase. As a rule the aurora has pulsating character. Johnstone (1983) and Davidson (1990) give detailed reviews of pulsating aurora properties. The typical size of pulsating patches of aurora is $20-100 \mathrm{~km}$. But sometimes the patches can be extended by hundreds kilometres in longitude (Johnstone, 1983). The characteristic period of auroral pulsations lies in the interval from fractions of a second to tens and even hundreds of seconds. Frequently the time of "turn-on" and "turnoff" of a patch is much smaller than its duration (Omholt and Berger, 1967). As a consequence, the luminosity of the aurora patch varies essentially non-sinusoidally. The pulsating auroras are induced by fluxes of energetic electrons precipitating into the ionosphere. Direct rocket measurements of pulsating fluxes show that the energy of precipitating electrons lies in the interval from several $\mathrm{keV}$ to one hundred and more $\mathrm{keV}$. Usually the ratio of fluxes in a maximum and a minimum of pulsations increases with energy, reaching $100 \%$ for energy above $50 \mathrm{keV}$ (Sandahl et al., 1980). The electron precipitations are observed from the midnight to the noon sector. During quiet geomagnetic periods a lo- cal maximum of the precipitation lies in the postdawn sector. During disturbed times the local maximum generally occurs in the predawn sector (Østgaard et al., 2000). The time delay of this morning precipitation relative to the substorm onset strongly indicates that this localised maximum is caused by electrons injected in the midnight sector and drifting into a region in the dawnside magnetosphere where some mechanism effectively scatters the electrons into the loss cone. The dawn local maximum is not observed in the precipitation of lower energy electrons, indicating that this precipitation consists mainly of energetic electrons (Østgaard et al., 1999). As a rule, simultaneously with precipitations, the bursts of ELF and VLF emissions are detected on the ground or on board a satellite. In particular, Isenberg et al. (1982), on the basis of a five-day observation interval, found that every encounter with injected clouds of energetic electrons was accompanied by chorus activity. They concluded that the dawnside chorus was generated by substorm-injected anisotropic clouds of electrons, with energies between 10 and $100 \mathrm{keV}$.

A theoretical analysis of particle precipitation from radiation belts due to the cyclotron interaction of VLF waves and energetic particles, based on a system of self-consistent equations of the plasma quasilinear theory (Vedenov et al., 1962), has been first applied by Andronov and Trakhtengerts (1964), and Kennel and Petschek (1966). Furthermore, some models of the cyclotron interaction were proposed for explaining the pulsating character of particle precipitation in the morning sector. Coroniti and Kennel (1979) analysed the possibility of the modulation of particle precipitation and VLF emissions by an external hydromagnetic wave. Davidson $(1979,1986)$ considered relaxation oscillations of the magnetospheric cyclotron maser. In such a model the system loses the equilibrium state due to pulsed injection of energetic particles. Under certain conditions the return of the system to a stable state looks like damped quasi-periodic oscillations. Bespalov (1981) has first shown that a magnetospheric cyclotron maser can have an auto-oscillating operation mode in the presence of a steady source of energetic electrons. Trakhtengerts et al. (1986) have offered a model of the flow magnetospheric cyclotron maser. In this model, a steady source of energetic electrons is provided by the drift of energetic electrons with initial transverse anisotropy of the pitch-angle distribution through a duct with enhanced cold plasma density, which serves as a resonance cavity. Pulsations with rather small $(\sim 10$-s) periods were explained by taking into account the self-consistent variation in the emission spectrum, due to the distortion of the particle distribution function. Demekhov et al. (1998) analysed the impulsive particle precipitation from the drifting electron cloud, taking into account the slow (compared with pulsation periods) variations of the energetic electron anisotropy and flux.

In the present paper we analyse in detail a case study of multisatellite observations of the drifting substorm electron cloud in the morning sector on 17 December 1990. During the event, ground-based photometric measurements of the 

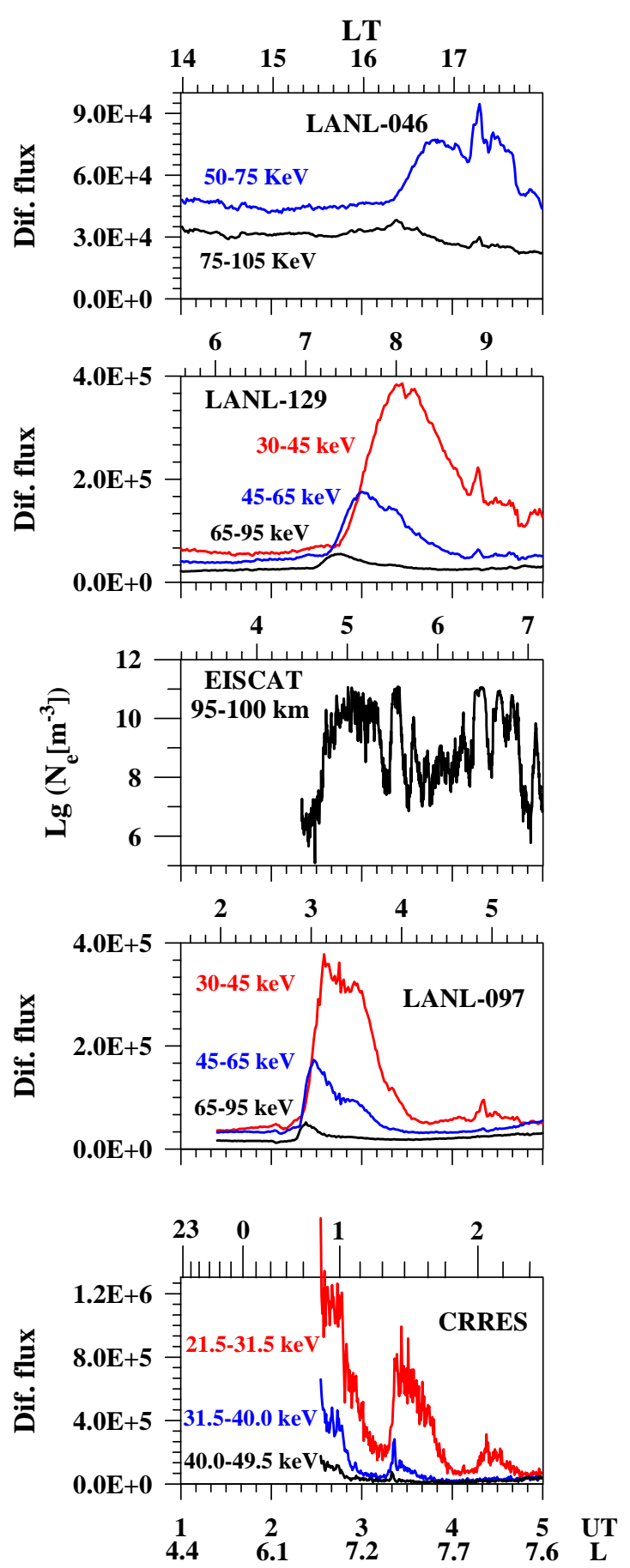

Fig. 1. Omnidirectional differential fluxes of electrons (in $\mathrm{cm}^{-2} \mathrm{~s}^{-1} \mathrm{sr}^{-1} \mathrm{keV}^{-1}$ ) in several energy channels, obtained on board the CRRES satellite and three geostationary LANL satellites spaced in longitude, and also the ionospheric electron density within the altitude range from 95 to $100 \mathrm{~km}$, observed by the EISCAT UHF radar, versus the universal time UT (bottom horizontal axis) and local time LT (upper horizontal axis). For the CRRES satellite its L-shell is also shown. auroral luminosity were performed, as well as measurements of the VLF emissions in the $0.3-10-\mathrm{kHz}$ frequency range and the electron density in the E-region (measured by the EISCAT UHF-radar). The detailed description of ground observations during the event development is given by Nygrén et al. (1992) and Manninen et al. (1996). In contrast to the statistical analysis by Åsnes et al. (2005), where the main focus was on the investigation of the dependence of energetic electron cloud properties on the longitudinal distance from the injection region, we compare the characteristics of one cloud observed by several satellites spaced in longitude. The flux values detected by one geostationary satellite in certain energy channels and different pitch-angle sectors are traced to longitudes of other satellites and to the longitude of the region of the observed particle precipitation. A comparison of results of the flux tracing and flux levels measured by satellites shows in which MLT sector the particle motion is described by the simple model of the magnetic drift. Comparison of the results of flux tracing to the longitude of the EISCAT UHF-radar and the character of ionospheric concentration variations allows us to analyse how the regime of particle precipitations depends on the flux levels in the drifting electron cloud. This, in turn, allows us to argue that the observed precipitation can, indeed, be explained by cyclotron interaction of energetic particles with VLF emissions.

\section{Analysed event and instrumentation}

We analyse the time interval from 01:00 until 05:30 UT of 17 December 1990. During this time interval the EISCAT UHF radar (inv. lat. $66.4^{\circ} \mathrm{N}$, inv. long. $103.3^{\circ} \mathrm{E}$ ) observed two series of electron density increases in the E-region (Nygrén et al., 1992; Manninen et al.,1996), following two small magnetic substorms developing on a quiet geomagnetic background. Simultaneous photometer observations verified that the phenomenon was caused by short impulsive particle precipitations having durations of about $4 \mathrm{~s}$ (Nygrén et al., 1992). The first sequence of precipitation pulses, which is our interest, lasted about $40 \mathrm{~min}$ from 02:30 until 03:10 UT. The series contained about twenty pulses of precipitation and was observed $20 \mathrm{~min}$ after the start of the substorm expansion phase (02:10 UT), developed in the midnight sector. The average period between the pulses was of the order of $100 \mathrm{~s}$ and the pulse duration was much shorter. The characteristic energy of precipitating electrons, as estimated from the height of the electron density maximum, is about $30 \mathrm{keV}$. Simultaneous VLF observations were carried out in Lovozero (inv. lat. $64.1^{\circ} \mathrm{N}$, inv. long. $114.7^{\circ} \mathrm{E}$ ) and Sodankylä (inv. lat. $63.9^{\circ} \mathrm{N}$, inv. long. $107.1^{\circ} \mathrm{E}$ ). During the event, an increase in the VLF chorus activity in the $0.3-3-\mathrm{kHz}$ range was observed; the activity was limited to frequencies below $1 \mathrm{kHz}$.

The EISCAT UHF radar carried out the measurements of the ionospheric concentration with the temporal resolution of $0.2 \mathrm{~s}$ and the spatial resolution of $1.05 \mathrm{~km}$ in the altitude 
range from $75 \mathrm{~km}$ to $145 \mathrm{~km}$. The radar beam was directed along the geomagnetic field line. Figure 1 shows 10 -s mean values of the ionospheric electron concentration in the altitude range from 95 to $100 \mathrm{~km}$.

We use the CRRES satellite data and particle measurements from three longitudinally spaced geostationary LANL satellites.

The CRRES satellite had an elliptical orbit with the perigee and apogee of $358 \mathrm{~km}$ and $33584 \mathrm{~km}$, respectively. The angle between the satellite orbit and equatorial planes is $18.1^{\circ}$. Johnson and Kierein (1992) give a detailed specification of the spacecraft mission and instruments. Until 02:31 UT a gap exists in the CRRES data. The satellite reached the apogee around 04:40 UT. In the time interval from 02:32 until 05:00 UT the CRRES is at magnetic latitudes of $25-23^{\circ}$ and at L-shells of $6.7-7.8$, i.e. it was projected onto the equator a little farther than the geostationary orbit. During the analysed interval the satellite is located in the midnight sector (00:50-02:40 MLT) near the substorm injection region. We use differential electron fluxes measured by the MEB (Medium Electrons $B$ ) spectrometer (Korth et al., 1992). The detector measures differential electron fluxes in 14 energy ranges from 21.5 to $285.0 \mathrm{keV}$, but we use only the data of four "low-energy" channels 21.5-31.5, 31.5$40.0,40.0-49.5$, and $49.5-59.0 \mathrm{keV}$. We use two data sets. One set is the level of differential electron fluxes across a magnetic field, at $85-95^{\circ}$ pitch-angles with a 30 s (one spin) time resolution. The second data set is the differential electron flux in 19 pitch-angle bins from $0^{\circ}$ to $180^{\circ}$, with a $10^{\circ}$ view angle. Thus, here a complete pitch-angle distribution of electrons is measured. For these data we have a 1-min time resolution. On the CRRES satellite the magnetic field components with temporal resolution of $5 \mathrm{~s}$ were measured, too.

We also use the particle measurements from three geostationary LANL satellites: 1987-097, 1984-129, and 1989046. At 01:00 UT their longitudes were at 01:35, 05:38, and 13:52 MLT, respectively. The detailed description of the CPA instrument measuring the energetic particle fluxes on board LANL-097 and LANL-129 is given by Higbie et al. (1978) and the SOPA instrument on board the LANL-046 satellite is described by Belian et al. (1992). The LANL-097 and LANL-129 satellites had particle detectors with identical energy levels. We use the data of the LoE detector subsystem measuring the electron flux in the "low-energy" range. The nominal energy levels of the LoE detector are 30, 45, $65,95,140$, and $200 \mathrm{keV}$, with an upper-energy cut off of $300 \mathrm{keV}$. From here it is possible to determine differential electron fluxes in the 30-45, 45-65, and 65-95 keV energy ranges. For these two satellites, the fluxes in three directions (for pitch angles $0 \pm 10^{\circ}, 90 \pm 10^{\circ}$, and $180 \pm 10^{\circ}$ ) and also an omnidirectional flux are calculated. In addition, according to the measured pitch-angle distribution of electrons with energy 30-300 keV, zero-, first-, second-, and fourthorder harmonics of the Legendre polynomial expansion are computed. As is well known, normalised first-, second-, and fourth-order harmonics of the Legendre polynomial, i.e. the ratio of this harmonic component to the zero-order component, are

$C_{1}=\cos \alpha_{0}$,

$C_{2}=1.5 \cdot \cos ^{2} \alpha_{0}-0.5$,

$C_{4}=4.375 \cdot \cos ^{4} \alpha_{0}-3.75 \cdot \cos ^{2} \alpha_{0}+0.375$,

where $\alpha_{0}$ is the equatorial pitch angle. Unfortunately, the LANL satellites do not have an on-board magnetometer. But the magnetic field direction is determined from the data on the particle pitch-angle distribution and its symmetry axis (Higbie et al., 1978). On the LANL-046 satellite only the omnidirectional electron flux in a few energy ranges is determined. In this study we use the differential electron flux in the 50-75 and 75-105 keV energy range only.

The omnidirectional differential electron fluxes in several lowest energy channels, measured on-board three geostationary satellites and by the MEB instrument on the CRRES satellite, are shown in Fig. 1. Three increases of energetic electron fluxes were observed. We investigate the first intensification, connected with the energetic electrons injected during a substorm expansion phase and drifting towards the morning side. According to ground magnetic data, a weak substorm $(\Delta H \sim 100 \mathrm{nT})$ occurred in the midnight sector (Manninen et al., 1996) in relation to the first injection. In Fig. 1 the time delay of the flux detection for higher MLT is well seen. For example, on the LANL-097 the maximum flux is observed at 02:32 UT, whereas on the LANL-046 it is observed at 03:50 UT. The spreading of the drifting particle clouds, caused mainly by a finite width of energy channels, is also seen. Note that the duration of the elevated flux, as well as flux levels are close to the statistical characteristics of the drifting clouds obtained by Åsnes et al. (2005).

\section{Method of flux longitudinal tracing}

As is known, the motion of a charged particle with energy of tens and hundreds $\mathrm{keV}$ in a geomagnetic field is composed of the fast rotation around the magnetic field line, oscillations between mirror points, and a relatively slow drift across the magnetic field line caused by the field line curvature and radial gradient of the magnetic field. The direction of the transverse drift depends on the particle charge. From the night sector the electrons drift to the morning side, while protons drift to the evening side. We use the guiding centre approximation. For the analysis of the guiding centre motion it is necessary to know the magnetic field. Unfortunately, we cannot know the actual geomagnetic field in any point of space at any moment of time. Therefore, we have to use some model of the magnetic field. Calculating the motion of the guiding centre in the chosen magnetic field model and averaging over 
the bounce period, we can determine the drift velocity across the magnetic field.

The choice of the magnetic field model is determined by what problem is solved and what accuracy of the solution is needed. We investigate the evolution of the electron cloud injected into the vicinity of the geostationary orbit during the substorm expansion phase. For this purpose we will need to recalculate the fluxes detected by one geostationary satellite to the longitude of another geostationary satellite (or the satellite which is located near the geostationary orbit). The comparison of the actual measured and expected (calculated) fluxes can give information about the physical processes occurring during the longitudinal drift. The accuracy of our calculations is obviously limited by many factors. First, we have no information on the depth of the substorm injection and its radial profile. However, this question is extremely important if a realistic magnetosphere model is used. Second, we do not have exhaustive information about the spectrum of drifting particles, since the particle detectors have a finite energy width. Third, we do not know the actual distribution of the magnetospheric electric field, and it can noticeably influence the motion of particles with energy of tens of keV. Fourth, we know rather crudely the pitch-angle distribution of drifting particles. Fifth, we do not know the pitch-angle diffusion coefficient. As a consequence, we can only estimate the change in the particle distribution caused by the diffusion on pitch angles. This question is important for comparison of omnidirectional fluxes because the drift velocity depends on the pitch angle. So, there are a lot of factors contributing to a noticeable inaccuracy in our calculations. Therefore, we consider using a simple dipole magnetic field model as a legitimate decision, especially since the geomagnetic activity was low for the analysed interval. The use of a more realistic model of magnetospheric fields in our specific case seems unjustified since it can produce an illusion of an accuracy which is too high for the calculations.

The approximation of the dipole magnetic field, which we shall use below, noticeably simplifies the calculations of particle drift velocity. However, it is necessary to understand clearly what possible effects are excluded from consideration in such a crude model. Shukhtina and Sergeev (1991) investigated the longitudinal drift of energetic particles near the geostationary orbit using the T-89 model (Tsyganenko, 1989). Calculations concerned to protons with $100 \mathrm{keV}$ energy (obviously, for such an energy it is possible to neglect the influence of the magnetospheric electric field). It has been noted that the energetic particles with small pitchangles move towards the Earth under the drift from the midnight sector to the noon sector, whereas near-equatorial particles move away the Earth. Even at the moderate geomagnetic activity $\left(K_{p}=3\right)$ the radial divergence of particles with different pitch angles, starting from one point of the geostationary orbit at the midnight meridian, can reach $2-4 R_{E}$. The divergence takes place even if the activity is low. For example, at $K_{p}=0$ the proton with a $90^{\circ}$ pitch-angle, starting at the mid- night meridian at the distance of $6.0 R_{E}$, crosses the noon meridian at the distance of $6.6 R_{E}$. Thus, if we are near the inner boundary of the injection region, this effect can essentially influence the flux levels measured by satellites spaced in longitude. The dependence of the drift velocity on longitude was also noted by Shukhtina and Sergeev (1991). But under moderate geomagnetic activity this effect is weak. For example, a proton with energy of $100 \mathrm{keV}$ and $90^{\circ}$ pitchangle, touching the geostationary orbit at 00:00 MLT, has the drift period of 65.4 min according to the T- 89 model. If the contact takes place at 12:00 MLT, the drift period is $67.2 \mathrm{~min}$. For the dipole field, the drift period is $65.5 \mathrm{~min}$. Thus, this effect does not lead to a noticeable inaccuracy in our calculations.

Even in the dipole magnetic field the rigorous expression for particle drift velocity has a rather intricate form. Therefore, it is useful to take advantage of the approximating equation proposed by Hamlin et al. (1961):

$$
\left\langle V_{d r}\right\rangle=9.4 \cdot \frac{E}{B_{0} \cdot R_{0}} \cdot\left(0.35+0.15 \cdot \sin \alpha_{0}\right) .
$$

Here $\left\langle V_{d r}\right\rangle$ is the longitudinal drift velocity in $\mathrm{m} / \mathrm{s}$ averaged over the oscillations between mirror points, $E$ and $\alpha_{0}$ are the particle energy (in keV) and equatorial pitch-angle, $B_{0}$ is the magnetic field (in Gauss) on the magnetic equator, and $R_{0}$ is the distance (in terrestrial radii) at which the magnetic field line crosses the magnetic equator. The sign of the particle charge defines the drift direction.

Approximation (1) with sufficient accuracy describes the longitudinal drift in the dipole magnetic field. It gives the noticeable inaccuracy only for small pitch-angles. In particular, Eq. (1) does not consider the precipitation of particles from the loss cone. Hence, in the case of the geostationary orbit it can be used only on pitch angles of $\alpha_{0} \geq 2.5^{\circ}$.

So, for the longitudinal tracing we assume that the magnetic field is the dipole one and use approximation (1). The magnetospheric electric field is not taken into account. At the tracing we suppose that the drifting electrons do not interact with either themselves or the VLF emissions.

A real satellite-borne detector measures the particle fluxes in some interval of energy, i.e. is practically an integral detector. As a consequence, the drift velocities for particles measured even in one energy channel can differ greatly. For example, according to Eq. (1), the drift velocity changes by a factor of 1.5 as the energy changes from 30 to $45 \mathrm{keV}$ (the first energy channel on the LANL-097 and LANL-129 satellites). Hence, for the longitudinal tracing it is necessary to know the particle fluxes for any energy from the analysed range, i.e. to know the spectrum of drifting particles.

Cayton et al. (1989) came to the conclusion that the spectrum of energetic electrons $(30-2000 \mathrm{keV})$ at the geosynchronous orbit is well described by two distinct Maxwellian components - "hard" (300-2000 keV) and "soft" (30$300 \mathrm{keV}$ ), which are fully parameterised by their densities 

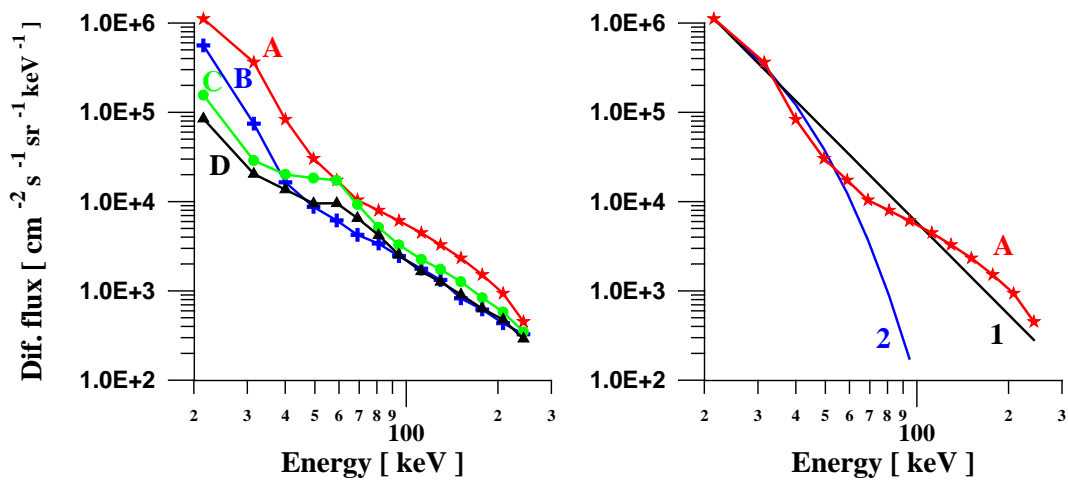

Fig. 2. On the left panel the dependence of differential fluxes on energy is shown in double logarithmic scale. Spectra of the omnidirectional differential fluxes observed by CRRES averaged over four time intervals, 02:33-02:47, 03:20-03:50, 04:15-04:45, and 04:00-04:15 UT, are denoted by $A, B, C$, and $D$, respectively. On the right panel the first spectrum $(A)$ is fitted by the power-law and Maxwellian functions, denoted by 1 and 2, respectively. The almost linear dependence suggests that the spectrum is defined by a power law, and is not Maxwellian.

and temperatures. The parameters of the "soft" electron component are determined by the intensity of substorm injection. On the other hand, if the average energy of particle distribution is not fixed (e.g., it increases and decreases due to the interaction with waves), but the average order of magnitude of the energy is constant, the particle distribution is described by the $\kappa$ function (Collier, 2004). As known, the high energy tail of the $\kappa$ distribution is described well by a power law:

$j_{\text {dif }}=C \cdot E^{-\beta}$.

Consideration of the average electron spectra of omnidirectional fluxes measured by the CRRES satellite (Fig. 2) demonstrates that, indeed, in our event the power law is more appropriate. In Fig. 2 the electron spectra for four time intervals $(A-D)$ are shown. Three intervals $(A-C)$ correspond to times of three injection detections. During the fourth interval $(D)$ the CRRES detects the background fluxes. Therefore, we shall use the power law (2) in the calculations. As is obvious from the left part of Fig. 2, the slope of the spectrum is almost independent of time and equals $\beta \sim 2.6$. But at the time of the first injection the CRRES satellite was close to the midnight sector, and close to the injection region. During the longitudinal drift of injected electrons the shape of spectrum can vary because the drift velocity depends on energy. We assume that at any moment of time within the limits of any two adjacent energy channels, the spectrum is defined by a power law. Two channels are necessary to obtain two unknown parameters, namely, the amplitude $C$ and the spectral slope $\beta$. Certainly, the parameters $C$ and $\beta$ can be functions of time and the analysed energy range. Let quantities $j_{\text {dif1 }}$ and $j_{\text {dif } 2}$ be the electron differential fluxes in an energy range from $E_{1}$ to $E_{2}$ and from $E_{2}$ to $E_{3}$, respectively, measured by the satellite $A$. Then it is possible to write

$j_{\mathrm{dif} 1}(t)=\frac{C(t)}{1-\beta(t)} \cdot \frac{E_{2}^{1-\beta(t)}-E_{1}^{1-\beta(t)}}{E_{2}-E_{1}}$ $j_{\text {dif2 }}(t)=\frac{C(t)}{1-\beta(t)} \cdot \frac{E_{3}^{1-\beta(t)}-E_{2}^{1-\beta(t)}}{E_{3}-E_{2}}$.

From here parameters $C(t)$ and $\beta(t)$ are easily determined. Hence, at any moment we can calculate the fluxes of particles of any energy in the range from $E_{1}$ up to $E_{3}$ at the satellite A.

Let the same electron with energy $E$ and pitch-angle $\alpha_{0}$ be detected by the longitude spaced satellites $A$ and $B$ at time moments $t_{1}$ and $t_{2}$, respectively. We can determine the relation between $t_{1}$ and $t_{2}$. If the projections of satellites to the geomagnetic equator at time $t_{2}$ have magnetic local times $t_{M L T}^{A}$ and $t_{M L T}^{B}$, then at this moment the distance between the satellites (along an arc of geomagnetic equator) is

$S=\frac{\pi \cdot R}{12} \cdot\left(t_{M L T}^{B}-t_{M L T}^{A}\right)$.

On the geostationary orbit the longitudinal drift velocity of the electron with energy, for example, $30 \mathrm{keV}$, is more than six times greater than the satellite velocity. However, it is impossible to completely neglect the satellite motion. Therefore, the desired time $t_{2}$ can be found from the equation

$S+\int_{t_{1}}^{t_{2}} V_{s a t}^{A}(t) d t=\left\langle V_{d r}\left(E, \alpha_{0}\right)\right\rangle \cdot\left(t_{2}-t_{1}\right)$.

Here the distance $S$ and drift velocity $\left\langle V_{d r}\right\rangle$ are defined by Eqs. (4) and (1), respectively. The longitudinal projection of the satellite velocity $V_{\text {sat }}^{A}$ to the geomagnetic equator of the geostationary orbit is easily determined by known orbital parameters. Equation (5) enables us to trace both forward, in the drift direction, and backward, to a source. If $t_{M L T}^{A}<t_{M L T}^{B}$, we determine naturally that $t_{1}<t_{2}$, and vice versa. Generally Eq. (5) is solved numerically. If the satellite $A$ is geostationary one, we can consider with satisfactory accuracy that $V_{\text {sat }}^{A}(t)=$ const. Thus, we neglect the difference in 

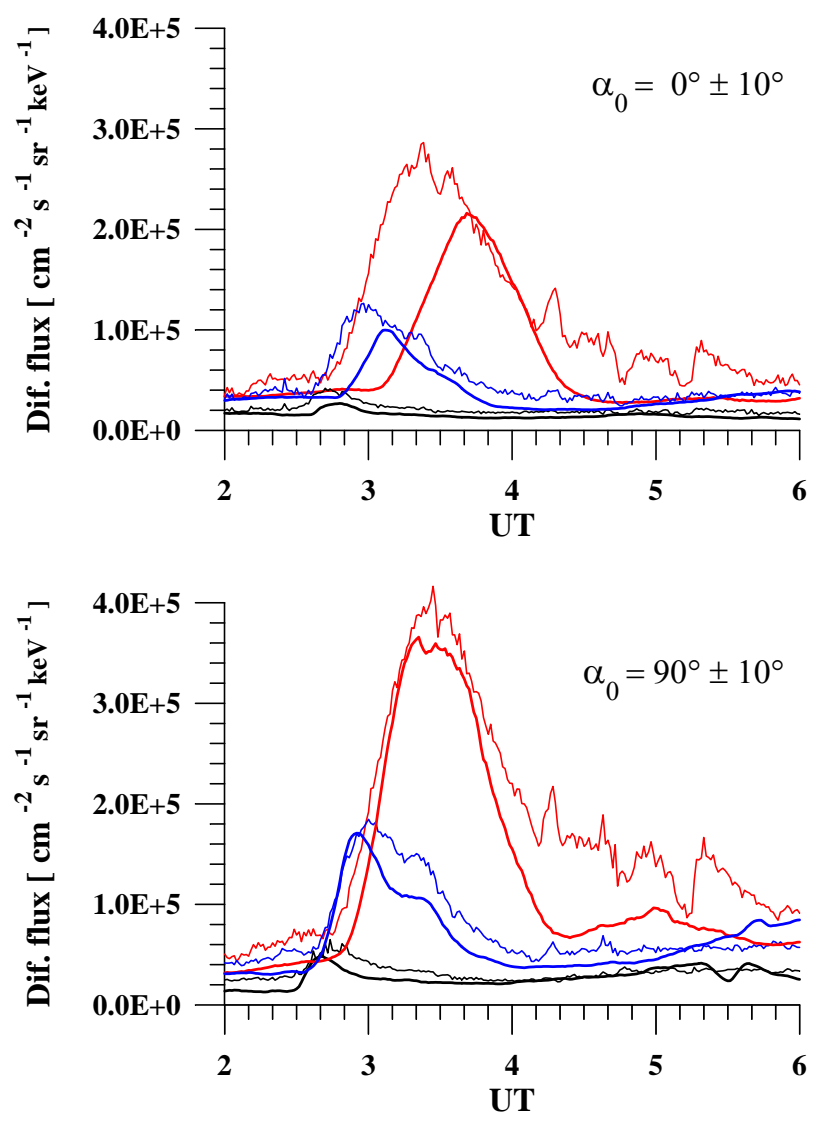

Fig. 3. Differential fluxes along (upper plot) and across (bottom plot) the geomagnetic field at $30-45,45-65$, and $65-95 \mathrm{keV}$ (the red, blue, and black line, respectively). The fluxes obtained by the LANL-129 satellite are shown by the thin line. Results of tracing the fluxes, observed by LANL-097, to the longitude of LANL-129 are shown by the bold line.

the projections of satellite velocity onto the geographical and geomagnetic equators. Then for the geostationary satellite $A$ Eq. (5) is simplified:

$t_{1}=t_{2}-\frac{t_{M L T}^{B}-t_{M L T}^{A}}{0.43 \cdot E \cdot\left(0.35+0.15 \cdot \sin \alpha_{0}\right)-1}$.

Now we can carry out the longitudinal tracing of fluxes from satellite $A$ to satellite $B$. Let at any moment $t_{2}$ be required to calculate the differential flux of electrons with the pitchangle $\alpha_{0}$ in the energy range from $E_{s}$ to $E_{f}$, which should be detected by the satellite $B$. For any energy $E_{a}$ within the range from $E_{s}$ to $E_{f}$, according to Eq. (6) for the geostationary satellite or Eq. (5) for the CRRES satellite, we define the drift time of electrons with energy $E_{a}$ and pitch-angle $\alpha_{0}$ between satellites, and, accordingly, the expected time $t_{1 a}$ of flux detection by the satellite $A$. According to Eqs. (3) we determine the spectral constants $C\left(t_{1 a}\right)$ and $\beta\left(t_{1 a}\right)$ for the flux detected by the satellite $A$ at the moment $t_{1 a}$. Then for a power spectrum (2) with known factors $C\left(t_{1 a}\right)$ and $\beta\left(t_{1 a}\right)$ the flux $j_{\text {dif }}\left(E_{a}, \alpha_{0}\right)$ of electrons with given energy which will be detected by the satellite $B$ at the moment $t_{2}$ is obtained. For another energy $E_{b}$, generally speaking, the expected time is $t_{1 b} \neq t_{1 a}$, accordingly, the spectral constants will also be different, i.e. $C\left(t_{1 a}\right) \neq C\left(t_{1 b}\right)$ and $\beta\left(t_{1 a}\right) \neq \beta\left(t_{1 b}\right)$. Integrating the flux over energy from $E_{s}$ to $E_{f}$ and dividing by the range width $E_{f}-E_{s}$, we calculate the differential flux in the range from $E_{s}$ to $E_{f}$ which should be detected by the satellite $B$ at any moment $t_{2}$. Comparing the calculated and measured flux values, we can estimate the correspondence between the actual motion of particles and the motion predicted by the simple model of the particle longitudinal drift in the dipole magnetic field.

\section{Results of longitudinal tracing}

\subsection{Projection of fluxes from LANL-097 to LANL-129}

The data from CRRES does not cover the beginning of the first substorm. Therefore, we choose LANL-097 as the "reference" satellite $A$, whose data are used for tracing, since of all the geostationary satellites it was the closest to the injection region. We trace the measured fluxes along and across the magnetic fields to LANL-129, along the line of the electron drift. These two satellites are spaced by approximately $60^{\circ}$ in longitude and, as mentioned above, have identical instruments. Figure 3 shows the results of the projection. Here are shown the differential directional fluxes along the magnetic field (pitch-angle $\alpha_{0}=0 \pm 10^{\circ}$ ) and across the magnetic field $\left(\alpha_{0}=90 \pm 10^{\circ}\right)$ in the first three energy channels, measured by the LANL-129 satellite, as well as the results of the tracing of fluxes measured by the LANL-097 at this longitude.

Let us analyse the obtained results. At first we consider the flux at perpendicular pitch angles. For all three energy channels we have quite a good agreement of the flux levels between the result of the projection and actual measurements. For the energy range of $30-45 \mathrm{keV}$ we have a good agreement for the detection time. For channels 45-65 and 65$95 \mathrm{keV}$ we obtain a good agreement for the start time of the cloud, whereas the trailing edge of the drifting cloud is observed approximately $10 \mathrm{~min}$ later than is expected from the tracing result. At small pitch-angles the situation is different. It is seen that calculated fluxes are in good accord with the detection time of the trailing edge of the drifting cloud. But an increase in the measured fluxes is observed significantly earlier than is expected from the tracing result (for example, by approximately half an hour for the first energy channel). The fluxes measured at small and perpendicular pitch angles vary almost synchronously, but the fluxes along the field are approximately $30 \%$ smaller.

The obtained results are described well by the following scheme. During the longitudinal drift, the particle separation in pitch angles (and energies) occurs. As a 
result the distribution function of electrons becomes strongly anisotropic and, as a consequence, unstable. Development of the cyclotron instability leads to a pitch-angle isotropisation. Therefore, the field-aligned fluxes vary synchronously with fluxes across the magnetic field. The energy of precipitating electrons in this event, estimated from the height of the electron density maximum on the EISCAT radar data, is of the order of $30 \mathrm{keV}$ (Manninen et al., 1996). During the cyclotron interaction the electrons with lower energy can give energy to VLF emissions, and high-energy electrons, on the contrary, can obtain energy from VLF emissions. As a consequence, electron fluxes at higher energy can increase.

To verify the possibility of the pitch-angle isotropisation during the longitudinal drift, we analyse, as far as the available data will allow us, the temporal evolution of the electron pitch-angle distribution at the LANL-097 and LANL129 satellites. In Fig. 4 the time dependence of the anisotropy parameter

$$
Q=j 90^{\circ} / j 0^{\circ}-1
$$

is shown. This parameter describes the ratio of fluxes across and along a magnetic field. For LANL-097 the flux ratio for the first three energy channels is shown. For LANL129 the behaviour of anisotropy in different channels is similar, therefore, only the dependence for the lower channel is shown. It is seen that on LANL-097 the anisotropy sharply increases when drifting substorm electrons are detected. The lower the energy, the higher the maximum anisotropy and the later it is observed. Indeed, the anisotropy is maximum at 03:20, 03:00, and 02:30 UT for the energy range of 30$45,45-65$, and $65-95 \mathrm{keV}$, respectively. On LANL-129 the anisotropy is noticeably lower and does not demonstrate considerable variations. This testifies that during the electron longitudinal drift the isotropisation of the pitch-angle distribution actually occurs somewhere between the satellites.

We plot the dependence of differential fluxes in the first energy channel on the ratio of fluxes at different pitch angles (Fig. 5). This allows us to compare our results with the statistical results of Åsnes et al. (2005). As is obvious from Fig. 5, on the LANL-097 satellite (03:00-04:00 MLT) the anisotropy remains low while the flux increases and subsequently increases toward the trailing part of the cloud. At the end of the cloud (03:41 UT) and at its beginning (02:22 UT) the anisotropy is almost the same. In accordance with the results of Åsnes et al. (2005) a clockwise sense of rotation in flux of cloud versus anisotropy is typical in the sector 02:00-04:00 MLT. The maximum anisotropy in our case is almost twice as much as the one obtained from the statistical analysis. This is probably caused by the fact that Åsnes et al. (2005) analysed the flux ratio at $90^{\circ} / 50^{\circ}$, whereas we used the flux ratio at $90^{\circ} / 0^{\circ}$. The duration of a cloud passage and the level of flux in our case are also typical for this MLT sector (Åsnes et al., 2005). On the LANL-129 satellite (07:0009:00 MLT) the anisotropy is more than twice as small as on the LANL-097. At the beginning and the end of the cloud
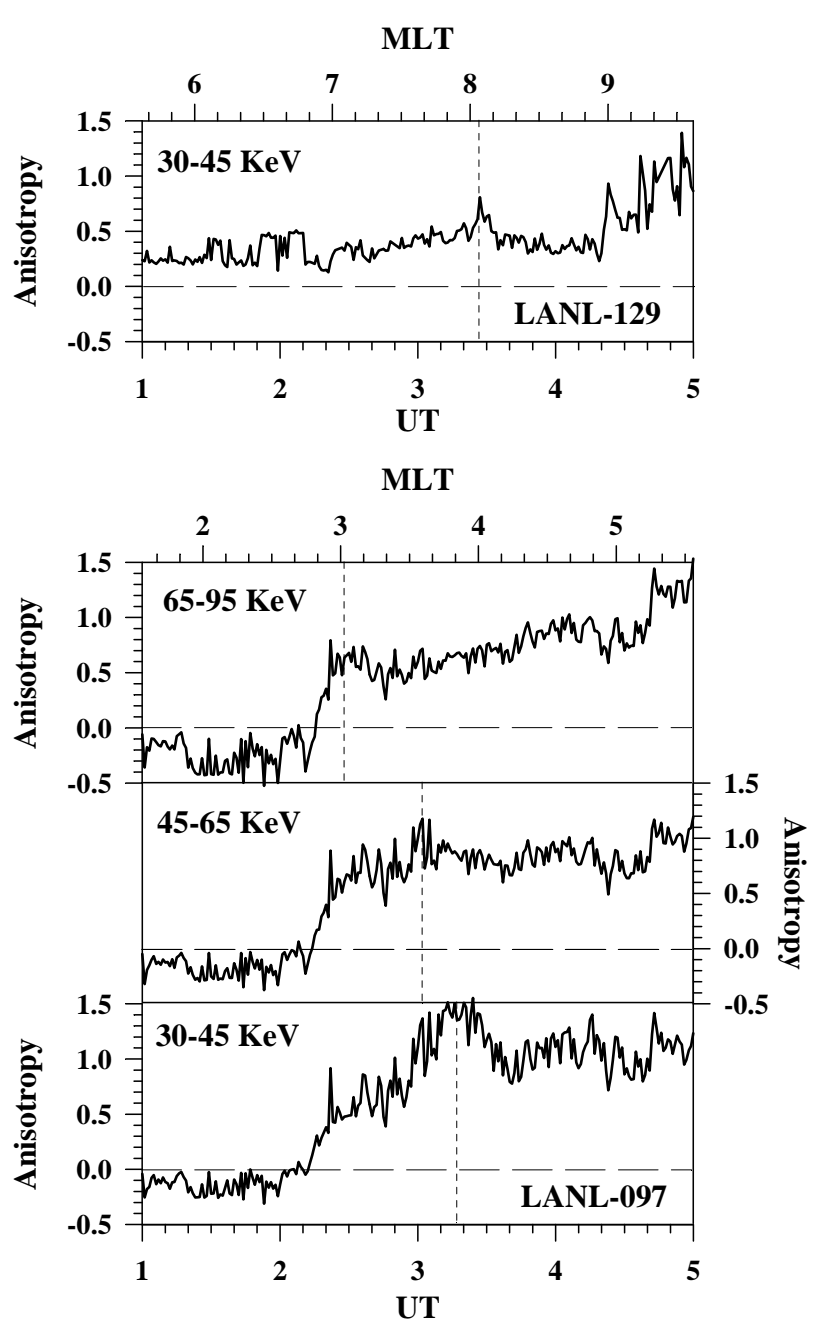

Fig. 4. Anisotropy of differential fluxes for three energy channels of the LANL-097 satellite and for the lower energy channel of the LANL-129 satellite.

the anisotropy is minimum on LANL-129 (on the very trailing brink of the cloud the anisotropy again increases, but this growth is caused by the new injection connected with a sudden impulse (see Yahnin et al., 1995). Anisotropy increases together with the increase in the flux in the cloud, reaching the maximum together with the flux. In contrast to early MLT, a rotation in flux versus anisotropy has a linear character. It also does not contradict the statistical results of Åsnes et al. (2005). In accordance with these results, in most cases, in a post-noon sector (15:00-17:00 MLT), a clockwise sense of rotation is replaced by an anticlockwise one.

Unfortunately, the ratio of fluxes across and along the magnetic field gives only a crude estimate of the pitch-angle distribution. Therefore we also use another possible method. Figure 6 shows even $\left(C_{2}\right.$ and $\left.C_{4}\right)$ harmonics of the Legendre polynomial expansion of the pitch-angle distribution function. The coefficient $C_{2}$ describes an oblongness of the 

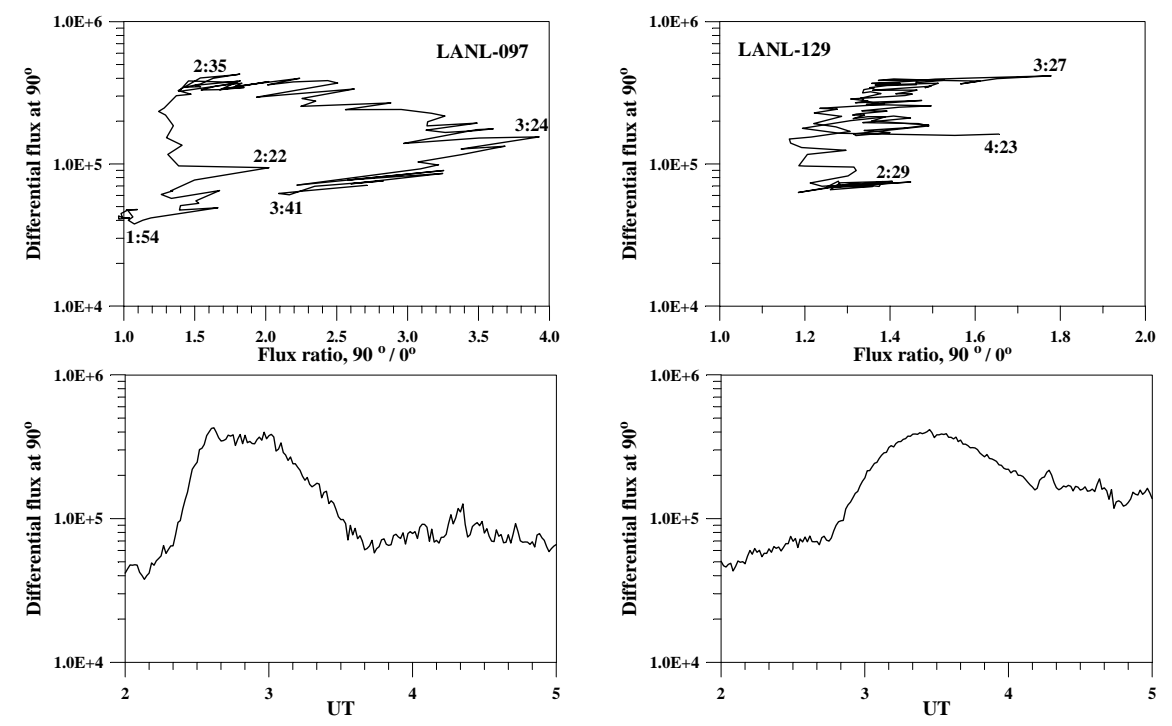

Fig. 5. The upper panels show the time trajectory in differential flux at $30-45 \mathrm{keV}$ across the magnetic field versus differential flux ratio at $90^{\circ} / 0^{\circ}$ observed at two geosynchronous satellites. Time labels correspond to the times of the flux detection. The bottom panels show the corresponding flux at the $90^{\circ}$ pitch angle.

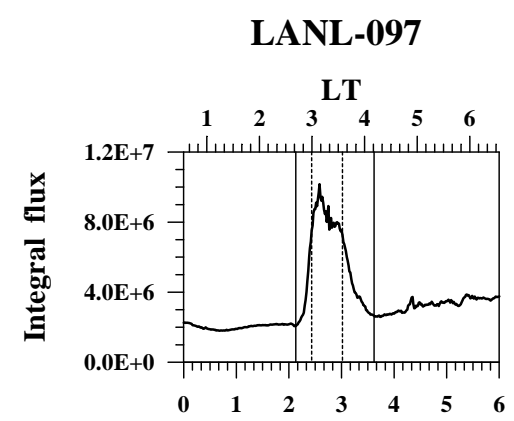

LANL-129
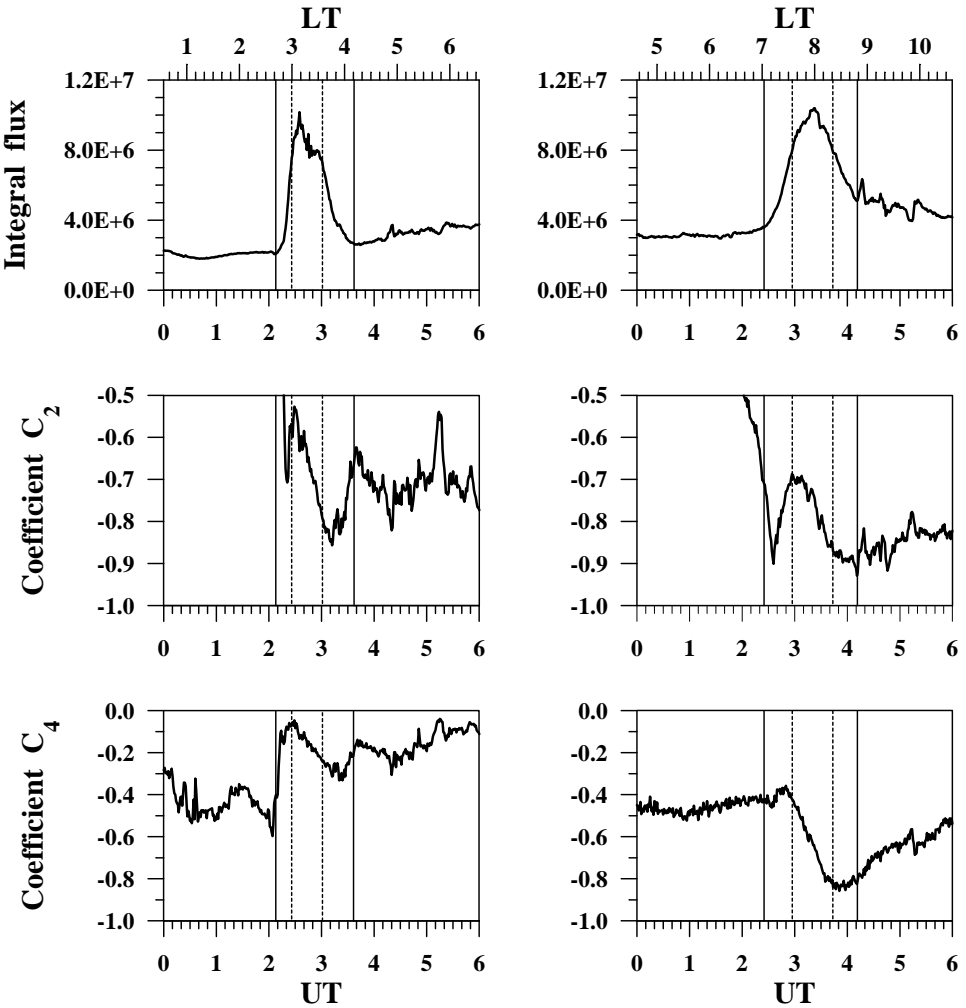

Fig. 6. Omnidirectional integral fluxes with energies above $30 \mathrm{keV}$ and the second- $\left(C_{2}\right)$ and fourth- $\left(C_{4}\right)$ order harmonics of the Legendre polynomial expansion of the pitch-angle distribution function obtained at two geosynchronous satellites. 
distribution function. $C_{2}<0$ indicates a "pancake-shaped" distribution (maximum flux at $90^{\circ}$ ) and $C_{2}>0$ indicates a "cigar-shaped" distribution (minimum flux at $90^{\circ}$ ). The coefficient $C_{4}$ describes the distribution of a "butterfly" type. If $C_{4}<0$, the distribution function has minima across and along a magnetic field, and maxima lie at $\alpha_{0} \approx 49^{\circ}$ and $\approx 131^{\circ}$. Change of the $C_{4}$ sign causes the places of the maxima and minima to swap. Vertical solid lines mark the moments of the beginning and end of the injected particle detection by a given satellite. Dashed lines select an interval of detection of the maximum fluxes. It is seen that the behaviour of coefficients has a similar character for both satellites. Both $C_{2}$ and $C_{4}$ there are negative. In the beginning of the event, $C_{4}$ is closest to zero and is almost constant, while $C_{2}$ reaches minimum after a sharp decrease. At this moment the distribution function is maximum "pancake-shaped", i.e. it has maximum anisotropy. Then $C_{2}$ increases a little, which testifies to a small decrease in anisotropy. After that, during the detection of the maximum fluxes, both coefficients decrease, becoming even more negative. This is equivalent to the expansion of the maximum at $90^{\circ}$. The maximum becomes broader. Now it is created by particles with the pitch angles lying in a wider range. At last, when detected fluxes start to decrease strongly, the coefficients $C_{2}$ and $C_{4}$ pass a range of the minimum values. As is obvious from Fig. 6, the coefficient $C_{2}$ on both satellites has similar values. However, on LANL-129 the $C_{4}$ coefficient is almost three times greater in absolute value than at LANL-097. This also testifies that the distribution function becomes more isotropic at later MLT. The expansion coefficients $C_{2}$ and $C_{4}$ bear qualitative information on the shape of the actual pitch-angle distribution. When the injected particles are detected by LANL-097 the distribution function is "pancake-shaped". On LANL-129 the distribution is close to isotropic in the interval of pitchangles from $50^{\circ}$ to $130^{\circ}$, having even a weakly pronounced minimum at $90^{\circ}$.

4.2 Projection of fluxes from LANL-097 to the longitude of the EISCAT radar

During the experiment of 17 December 1990 the beam of the EISCAT radar was directed along the geomagnetic field line. The difference between the longitudes of the point where this field line crosses the geomagnetic equator and the LANL097 satellite is about $24^{\circ}$ (or 01:36 MLT). The tracing results of the fluxes detected by LANL-097 on the longitude of the EISCAT radar are shown in Fig. 7A for fluxes along the field $\left(\alpha_{0}=0^{\circ}\right)$, across the field $\left(\alpha_{0}=90^{\circ}\right)$, and also omnidirectional fluxes $\left(j_{o m n i}\right)$ in the energy range from 30 to $50 \mathrm{keV}$. We calculate the expected omnidirectional fluxes in the following way. For simplicity we suppose that the directed flux on LANL-097 linearly depends on the pitch angle, i.e.

$j\left(\alpha_{0}\right)=j_{0^{\circ}} \cdot\left(1+Q \cdot \frac{\alpha_{0}}{90^{\circ}}\right)$,
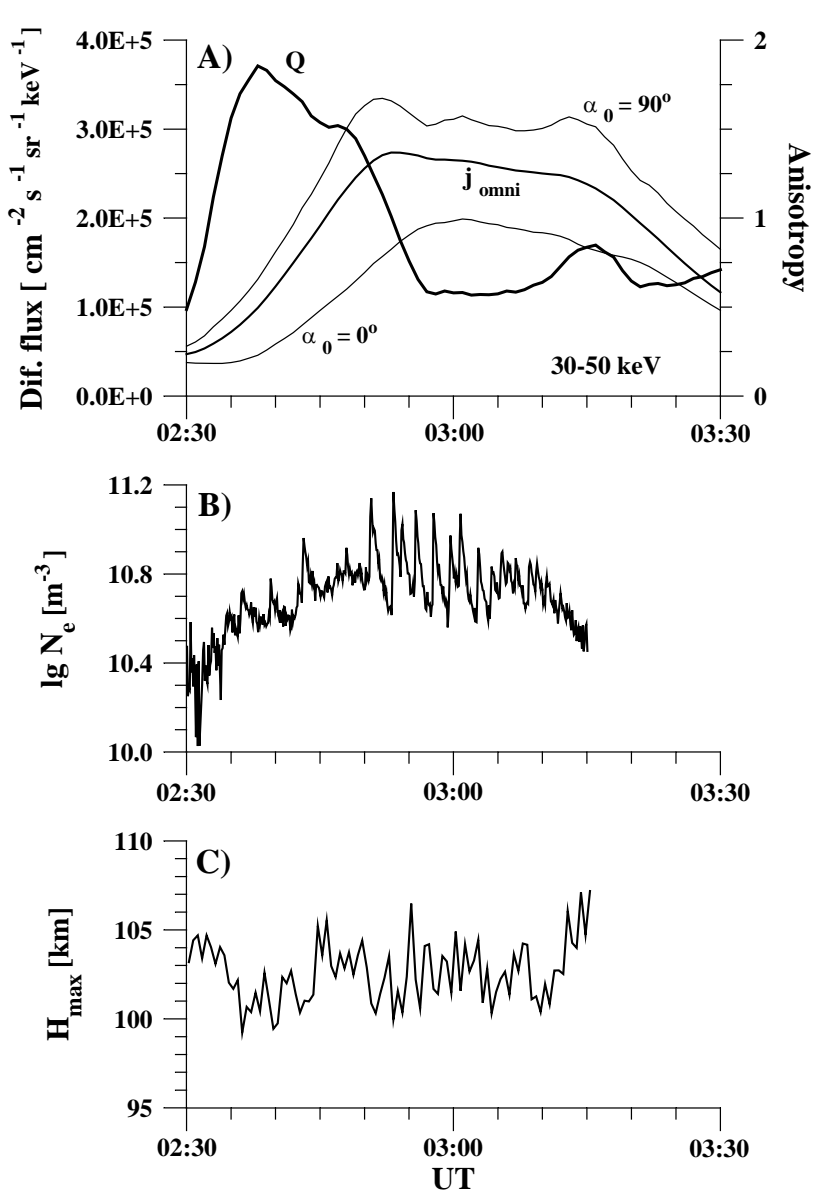

Fig. 7. (A) Results of tracing of the field-aligned $\left(\alpha_{0}=0^{\circ}\right)$, perpendicular $\left(\alpha_{0}=90^{\circ}\right)$, and omnidirectional fluxes obtained by the LANL-097 satellite to the longitude of the EISCAT UHF radar. The expected anisotropy of the traced flux is shown by the bold line. (B) The 6-s average of the maximum ionospheric electron density within the altitude range of $85-115 \mathrm{~km}$, observed by the EISCAT radar. (C) The 30-s average of the height at which the ionospheric electron density reached a maximum.

where the anisotropy parameter $Q$ is defined by Eq. (7) and is a function of time (see Fig. 4), and the equatorial pitchangle $\alpha_{0}$ is measured in degrees. Now it is possible to obtain the relation between the directed and omnidirectional fluxes

$j\left(\alpha_{0}\right)=j_{\mathrm{omni}} \cdot \frac{1+Q \cdot \frac{\alpha_{0}}{90^{\circ}}}{1+Q \cdot \frac{2}{\pi}}$.

Equations (8)-(9) are valid for $\alpha_{0}$ within the $0-90^{\circ}$ pitchangle range, but we assume a symmetry of flux in the 0 90 and $90-180^{\circ}$ pitch-angle ranges. Note that Eq. (9) is not a very rough approximation. Indeed, while the LANL-097 satellite detected the drifting electron cloud it measured on average $Q \approx 0.9, j_{90^{\circ}} \approx 1.2 \cdot j_{\text {omni }}$, and $j_{0^{\circ}} \approx 0.6 \cdot j_{\text {omni }}$, which is well in accord with Eq. (9). Knowing the values of $j_{\text {omni }}$ and $Q$ and using Eq. (9), we can calculate the directed flux 

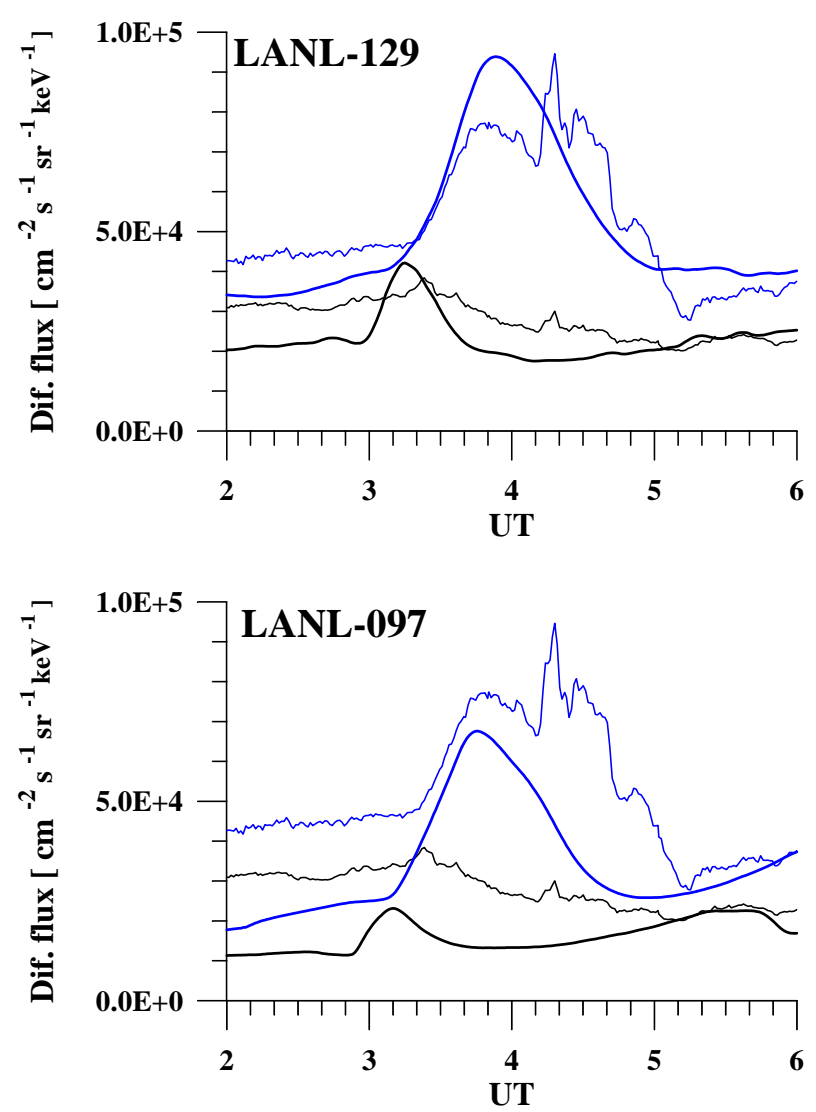

Fig. 8. Omnidirectional differential fluxes at $50-75$ and $75-105 \mathrm{keV}$ (the blue and black line, respectively). The fluxes obtained onboard the LANL-046 satellite are shown by the thin line. Results of tracing of the fluxes detected by the LANL-129 (top) and LANL-097 (bottom) satellites to the longitude of the LANL-046 satellite are shown by the bold line.

on LANL-097 for any pitch angle and any moment of time $t$. Projecting these fluxes on the longitude of the EISCAT radar and integrating fluxes over pitch angle, we receive expected omnidirectional fluxes of $j_{\text {omni }}$ at the longitude of the EISCAT radar. In Fig. 7A the expected dependence on time of the anisotropy parameter $Q$ defined by Eq. (7) is also shown. It is seen that transverse anisotropy should be maximum at the beginning of a drifting particle cloud.

Using the EISCAT radar data with 1-s time averaging, we obtained a maximum ionospheric electron density in the altitude range of $85-115 \mathrm{~km}$ and the height at which this density was reached. The received maximum density averaged over a 6-s interval is shown in Fig. 7B. It is seen that the electron density increases exactly during the passage of the drifting particle cloud. During the expected detection of the maximum fluxes of drifting particles, the increase in the ionospheric electron density has clearly expressed a pulsating character. The curve is plotted only to 03:15 UT, so that it excludes the contribution of the second injection detected at
03:19-03:26 UT and caused by another reason. Figure 7C shows a 30 -s averaged height $H_{\max }$ at which the ionospheric electron density is maximum. It is seen that the height varies little during the event (in the mean, $H_{\max } \approx 103 \mathrm{~km}$ ), which indicates that the energy of the precipitation electrons varies little during the event. From the altitude profile of the ionospheric density, one cannot unambiguously retrieve the spectrum and the flux of precipitated particles. Therefore, the energy range from 30 to $50 \mathrm{keV}$ chosen for tracing is an approximate one. The precipitated electrons with such energies cause the increase in ionospheric density at altitudes above $90 \mathrm{~km}$. If we consider more energetic particles, then the expected fluxes on the longitude of the EISCAT radar will start increasing a few minutes earlier. The account of low energy particles, on the contrary, will lead to a little later start in the flux decrease, but it will not affect the basic result in which the EISCAT radar detected the increase in the ionospheric electron density during the expected passage of the drifting electron cloud.

Below we try to estimate the spatial limits of the validity of the described method of longitudinal tracing.

\subsection{Projection of fluxes from LANL-097 and LANL-129 to LANL-046}

The LANL-097 and LANL-046 satellites are spaced in longitude by $186^{\circ}$, i.e. are at a rather significant distance. The longitudinal distance between LANL-129 and LANL-046 is less by $\sim 60^{\circ}$ (i.e. $125.5^{\circ}$ ), but it is also large enough. Energy channels of the LANL-046 satellite differ from the channels of the other two satellites by both the channel width and the measured energy range. In addition, we have only data of the omnidirectional fluxes detected by LANL-046. All this naturally complicates the comparison of the data recorded by different satellites. However, we make such an attempt.

In Fig. 8 the results of tracing of the fluxes detected by LANL-097 and LANL-129 to the longitude of LANL-046 are shown. Calculations were performed for energy intervals of $50-75$ and $75-105 \mathrm{keV}$, which correspond to the range of the two lower energy channels of LANL-046. The tracing method of omnidirectional fluxes is similar to the one used above for the calculations of the expected flux at the longitude of the EISCAT radar. As is obvious from Fig. 8, the expected detection time of drifting electron fluxes in the energy range of 50-75 keV corresponds well to the actual time of the flux detection by LANL-046. A sharp increase in the detected fluxes at 04:18 UT occurs simultaneously in a wide longitudinal sector and is related to the magnetospheric compression, therefore, it is not reproduced in the modelling of the longitudinal drift in the geomagnetic field. The recorded background fluxes before the arrival of the drifting particle cloud are approximately twice the expected fluxes traced from the LANL-097 satellite and 1.4 times above those obtained by tracing from the closer LANL-129 satellite. In both cases the ratio between the actual and expected background 
fluxes monotonically decreases with time. These distinctions can be caused by the geomagnetic field's nondipole component, especially evident before a substorm expansion phase. For example, calculations by Åsnes et al. (2005), based on the Tsyganenko T96 model, show that the magnetic field strength on the geostationary orbit at the noon sector should be approximately twice the field in the midnight sector. During the substorm expansion phase the geomagnetic field should become more dipole-like. Perhaps for this reason the expected and recorded fluxes in the drifting particle cloud are close in value. The maximum flux detected by LANL-046 in the substorm electron cloud is only 1.1 times above the expected maximum flux as traced from LANL-097; this is a good correspondence. On the contrary, the expected fluxes as traced from LANL-129 are approximately 1.2 times above the actual fluxes. This can be caused by the fact that the chosen model of the pitch-angle dependence (Eq. 9) is unsuccessful for the LANL-129 satellite. Indeed, during the detection of the drifting electron cloud by LANL- 129 we have on average $Q \approx 0.4, j_{90^{\circ}} \approx j_{\text {omni }}$, and $j_{0^{\circ}} \approx 0.7 \cdot j_{\text {omni }}$. It is seen that calculations on Eq. (9) overestimate the fluxes both across the field $\left(j_{90^{\circ}}^{\bmod } \approx 1.1 \cdot j_{\text {omni }}\right)$ and along the field $\left(j_{0^{\circ}}^{\bmod } \approx 0.8 \cdot j_{\text {omni }}\right)$. Varying the model of the pitch-angle distribution, it is possible to achieve a better agreement between the modelled and actually detected flux for the LANL-046 satellite.

Thus, during weakly disturbed geomagnetic conditions the calculations in the dipole magnetic field approximation allow one to predict the time of detection and the flux level of drifting substorm electrons for rather large distances from the substorm injection region, down to the evening sector.

\subsection{Projection of fluxes from LANL-097 to CRRES}

Now let us consider the correspondence of fluxes at LANL097 and CRRES during the first substorm injections. At this time CRRES was approximately $35^{\circ}$ to the west of LANL097, i.e. closer to the injection region. As a consequence of the strongly elongated elliptical orbit of CRRES, the longitudinal distance between it and LANL-097 does not remain constant. We take this into account in our longitudinal tracing. At the same time CRRES is on an L-shell of 6.7-7.3, i.e. a little farther from the geostationary orbit. If we assume that the injection propagates earthward, the radial propagation time should also be taken into account. According to estimates of the propagation velocity by Reeves et al. (1996), this time correction does not exceed 3 min. Only the electron injection was detected by CRRES; the proton injection was absent. Due to a lack of CRRES data until 02:31 UT we do not know whether the injection was dispersionless or not. Thus, we can only affirm that the satellite is either in the eastern part of injection region (region 5, according to Birn et al., 1997) or slightly eastward of it.

Using the flux level of electrons with energy of $30-65 \mathrm{keV}$, detected by LANL-097, we determine the flux level in the energies ranges corresponding to the three first channels of CRRES, i.e. 21.5-31.5, 31.5-40.0, and 40.0-49.5 keV. Next, we trace the calculated fluxes to the longitude of CRRES. For tracing of omnidirectional fluxes, we use the same procedure as for the calculations of expected fluxes at the longitude of the EISCAT radar and LANL-046. The results of the tracing are shown in Fig. 9.

The level of fluxes measured on CRRES and traced from LANL-097 are in a satisfactory agreement, keeping in mind the numerous sources of calculation errors. Indeed, the energy from channels of CRRES differ from channels of LANL-097, both in the energy range and in their energy width. The energy range of the lower channel of CRRES is almost not covered by the ranges of the LANL-097 channels. Also, the omnidirectional flux measured by CRRES is formed only by particles having pitch angles from 0 to $\sim 44^{\circ}$ in the equatorial plane, because during the injection CRRES has a magnetic latitude of about $24^{\circ}$.

At the same time a significant inconsistency follows from the timing of the fluxes. At the position of CRRES the increase in backward-traced higher energy fluxes is delayed relative to the increase in the lower energy fluxes. In a simple model of pure magnetic drift it means that CRRES is well inside of the injection region. But even inside of the injection region (and of course eastward of it) the magnetic drift model, if applicable, should reproduce the trailing edge of the electron cloud. In reality, we see that the time difference between the registration of the trailing edge and that traced backward is sometimes more than $20 \mathrm{~min}$, and it varies with energy and pitch angle.

Thus, independent of the real position of the spacecraft (within or close to the injection region) the simple model of drift in the dipole magnetic field is inapplicable within the longitudinal sector of 01:00-03:00 MLT for the analysed energy range $(21.5-65 \mathrm{keV})$. It is possible to assume, that in this longitudinal sector, the drift velocity is significantly influenced by a nonstationary substorm-related electric field, similar to a transient localised azimuthal electric field considered in the models of Roederer and Hones (1974), Birn et al. (1998), Li et al. (2003), and Sarris and Li (2005).

\section{Impulses of the ionospheric electron density increase}

The increase in the ionospheric electron density during the passage of a drifting electron cloud has a pulsed character; this is seen well in Fig. 7B. Simultaneous photometer observations testify that these increases are caused by short (with duration about $4 \mathrm{~s}$ ) electron precipitations (Nygrén et al., 1992). As shown by Nygrén et al. (1992) the decay of the electron density between precipitation impulses has a characteristic time of $30-40 \mathrm{~s}$, and is due to recombination processes. On average, pulses of precipitation follow each other in $100 \mathrm{~s}$. Our analysis showed that the recurrence interval of pulsed precipitations changes continuously during the 

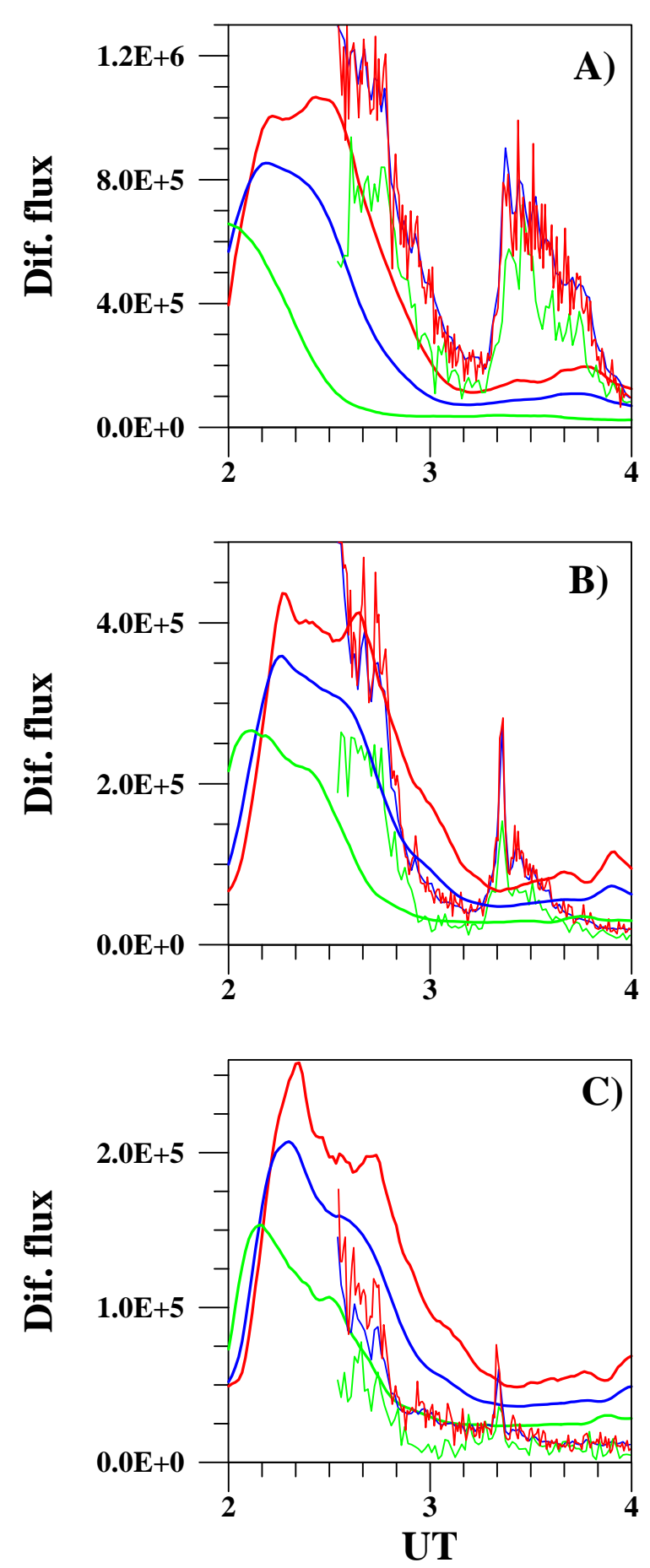

Fig. 9. Differential fluxes along (green) and across (red) the geomagnetic field and omnidirectional fluxes (blue) within the energy channels $21.5-31.5 \mathrm{keV}$ (A), 31.5-40.0 keV (B), and 40.0-49.5 keV (C). The fluxes detected by the CRRES satellite are shown by the thin line. Results of tracing of the fluxes, detected by the LANL097 satellite to the longitude of the CRRES satellite, are shown by the bold line. event. It is $\sim 100 \mathrm{~s}$ in the beginning of the event, reaches 200 $300 \mathrm{~s}$ in the middle, and at the end of the event it is $\sim 50 \mathrm{~s}$. As follows from Fig. 7C, the average height of the maximum of ionospheric electron density, as well as the average energy of precipitated particles, vary weakly during the event. We have separately analysed properties of pulses at growth and decreasing stages of the precipitation particle flux. For this purpose we have divided the ionospheric density increase pulses detected by the EISCAT radar into two groups. The first group is formed by nine pulses observed in the first half of the event when the intensity of pulses and the interval between them increased: 02:34:22, 02:36:06, 02:39:23, 02:42:31, 02:43:05, 02:50:40, 02:53:12, 02:54:09, and 02:55:43 UT. The second group is formed by seven pulses observed at the end of the event when the pulse intensity and the interval between them decreased: 02:57:43, 02:59:36, 03:00:43, 03:02:45, 03:04:14, 03:05:19, and 03:06:54 UT. To analyze the pulse properties we used the superposed epoch method. For each pulse the reference moment of time $t_{0}$ is the moment of observation of the maximum ionospheric density (the corresponding times are listed above). For each pulse, the average ionospheric density was determined for a two-minute interval centred at $t_{0}$; then this average level was subtracted. After that, the data was normalised in such a way that the amplitude of the pulse varied from -1 up to +1 . The averaged dynamics of the electron density (fast increase and slow decay) is shown in Fig. 10A.

We analyse how the height of the maximum of ionospheric electron density varies within a pulse separately for both pulse groups. The height of the $N_{e}$ maximum was averaged over two-minute intervals centred at $t_{0}$. Variations of the height of the $N_{e}$ maximum relative to this averaged value for the two groups of pulses are shown in Figs. 10B and 10C. For the first group of pulses one can see a clear decrease of the height during the precipitation burst (the red line), as well as a general decrease in the height on the time scale of tens of seconds (the blue line). For the second group of pulses the behaviour of the height of the $N_{e}$ maximum is very different. There are no significant changes in the height during the precipitation burst (the red line). On the larger time scale one can see the increase (decrease) in the height of the $N_{e}$ maximum before (after) the burst.

Since the pulse duration $(\sim 4 s)$ is smaller than the ionospheric recombination time scale $(\sim 30-40 \mathrm{~s})$, it is possible to assume that the height of the $N_{e}$ maximum during the precipitation burst is determined by the energy of the precipitated electrons. It is worth noting that just before the precipitation pulse the ionospheric electron density changes insignificantly, so that the height of the $N_{e}$ maximum is also determined by the energy of the precipitated electrons. However, just after the pulse the height of the $N_{e}$ maximum can depend as well on the recombination coefficient, which can be different at different altitudes. Thus, at least before and during the burst the change in the $N_{e}$ maximum height reflects the change in the precipitated particle energy. The higher the 
energy of the particles, the lower the maximum of $N_{e}$.

As it follows from the comparison of Figs. 10B and 10C, the properties of pulses at the growth stage and at the stage of decreasing precipitated electron flux are different. In the first group, the energy of precipitated particles increases during the burst, whereas at the end of the event this energy varies insignificantly. The energy of particles in the background precipitation before the burst increases (decreases) with time at the beginning (end) of the event. This can mean that there is a difference in the pulse formation mechanisms at the growth and decrease stages of the precipitated particle flux.

\section{Discussion}

According to the data of three geostationary satellites of the LANL series spaced in longitude and the CRRES satellite, the temporal and spatial evolution of a cloud of electrons with energies above $20-30 \mathrm{keV}$ drifting on the morning side has been analysed. Particles have been injected during the substorm in the midnight sector. The substorm developed on a quiet geomagnetic background. Our analysis has shown that in the longitudinal sector from 03:00 until, at least 09:00 MLT the drift of electrons with $90^{\circ}$ pitch angles is well reproduced by the simple model of longitudinal drift in the dipole magnetic field.

As is known, particles with different pitch angles have different drift velocities. Therefore, during the longitudinal motion the pitch-angle anisotropy in the drifting cloud of the injected particles should vary continuously. At some distance from the injection region at the beginning of the drifting cloud particles with $90^{\circ}$ pitch angles will start to dominate; accordingly, the transverse anisotropy will be high at the front of cloud, and this transverse anisotropy increases with an increase in the distance from the injection region. To the centre of a cloud, where the flux increases, the anisotropy should decrease. At the trailing edge of the drifting particle cloud the particles with small pitch angles should dominate. Such a pattern should be observed if there is no pitch-angle diffusion. However, it is known that the particle distribution function with the high transverse anisotropy is unstable with regard to the cyclotron instability. Therefore, as some critical anisotropy is reached, the electron-cyclotron interaction between the drifting high-energy electrons and VLF emissions should start. This leads to the generation of VLF emissions and particle diffusion on energies and pitch angles. As a consequence of this diffusion, the electrons are scattered into the loss cone and precipitate into the ionosphere, while the anisotropy decreases. Later on, the anisotropy can remain near the stability limit, i.e. the continuous growth of the anisotropy caused by the particle drift will be compensated by the weak or moderate pitch-angle diffusion. A similar schematic model is considered by many authors (e.g. Torkar et al., 1987; Åsnes et al., 2005). Our results agree with this
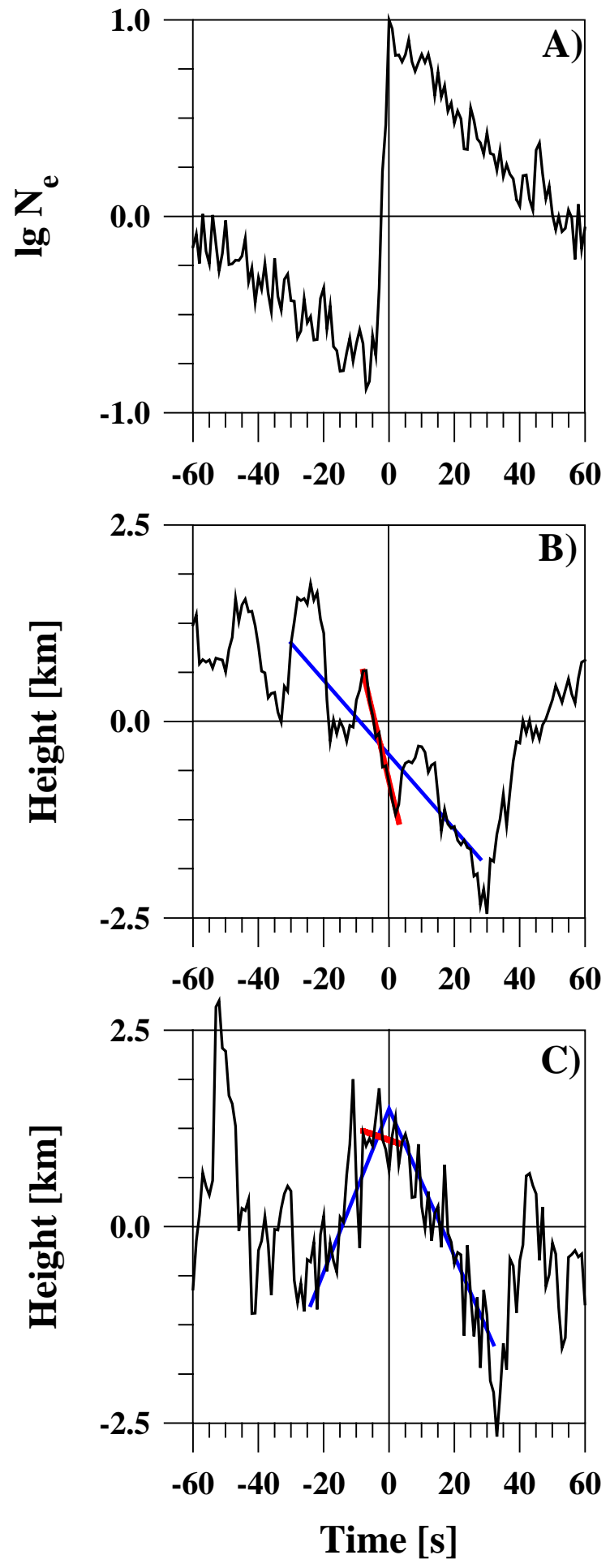

Fig. 10. (A) Change in the ionospheric electron density observed by the EISCAT radar during pulsed electron precipitations. (B) and (C) Change in the height of the ionospheric density maximum in the pulses observed in the beginning $(\mathrm{B})$ and at the end $(\mathrm{C})$ of the event. 
scheme. Indeed, at 08:00 MLT, already approximately after $70^{\circ}$ of the longitudinal drift, fluxes at small and perpendicular pitch angles varied almost synchronously. In other words, during the longitudinal drift the expected pitch-angle separation of electrons is absent. This just means that the distribution function of high-energy electrons becomes unstable during the drift. The cyclotron instability has led to pitch-angle isotropisation. Conforming to this assumption, approximately at $30^{\circ}$ of longitude from LANL-097 in the line of drift the EISCAT UHF radar has detected an increase in the ionospheric electron density $N_{e}$, having a maximum at heights $100-105 \mathrm{~km}$. The $N_{e}$ increase was observed at the estimated time of the drifting cloud passage of the longitude of the EISCAT radar measurements. Simultaneously, at the stations Lovozero $\left(67.97^{\circ} \mathrm{N}, 35.08^{\circ} \mathrm{E}\right)$ and Sodankylä $\left(67.51^{\circ} \mathrm{N}, 26.33^{\circ} \mathrm{E}\right)$, VLF emissions in the $0.3-1-\mathrm{kHz}$ frequency range were observed (Manninen et al., 1996). All this testifies to the development of the cyclotron instability leading to the generation of VLF waves and precipitation of energetic electrons caused by their scattering into the loss cone.

In our case the electron precipitation had a pulsed character. It is worth noting that a substorm activation typically consists of several one-minute elementary events with approximately 2-3-min repetition rate (e.g. Sergeev and Yahnin, 1979; Yahnin et al., 1983; Sergeev et al., 1986). This fine structure of the substorm has been resolved in the differential proton flux registered within a relatively narrow energy range ( $E=400-500 \mathrm{keV}, \Delta E / E \approx 0.2 ; \Delta E$ is energy range of the detector) on board the LANL spacecraft (Yahnin et al., 1990). Such periodicity is comparable with the average period of the impulsive electron density increases. But model calculations (not presented here) made for electrons measured by the low energy electron detector on board the LANL spacecraft evidence that during the longitudinal drift the fine structure of injected particles should smooth out quickly due to the drift velocity dispersion. Thus, the pulsed character of precipitations, observed in our case, cannot be related to the fine structure of the injection.

As is known, the pulsed precipitation and related pulsating aurora are typical phenomena for the morning sector during a substorm recovery phase. Thus, the mechanism of the cyclotron instability development in the morning sector after a substorm should provide a pulsating mode of electron precipitation. Some possible mechanisms have been described in the literature. Coroniti and Kennel (1970) considered the possibility of modulation of the VLF emissions, causing pitch-angle diffusion, by an external hydromagnetic wave. Magnetic pulsations in the $P c 1$ frequency range are, indeed, often detected together with pulsating aurora (Heacock and Hunsucker, 1977). Sato (1984) has given an example of a good correlation between periodic ( $T \sim 5.6 \mathrm{~s})$ VLF emissions and short-period magnetic pulsations observed in the Antarctica at Syowa station $(L \sim 6)$ under very quiet geomagnetic conditions. He assumed that the generation of magnetic pul- sations and VLF emissions occurs self-consistently. Periodic VLF emissions cause periodic electron precipitations. The precipitations increase the ionospheric conductivity in the Dand E-regions. This, in turn, leads to the generation of magnetic pulsations. Similar mechanisms assume the occurrence of a periodic smooth variation of precipitation particle fluxes, accompanied by the VLF emissions, mainly noise character. They can explain electron precipitations with a duration of about $5 \mathrm{~min}$ and the repetition period about $15 \mathrm{~min}$, which were also observed on 17 December 1990 from 04:15 UT. Simultaneously with those pulsations, periodic magnetic field variations were detected at the geostationary orbit (Manninen et al., 1996).

However, mechanisms of the pulsating aurora formation by an external hydromagnetic wave do not always work. In particular, such mechanisms cannot explain our short-pulsed precipitations with the high repetition period, observed after the substorm expansion phase. We also note that during this observation, mainly discrete, not noise-like, VLF emissions were detected. The long-period smooth magnetic pulsations, on the contrary, were not observed. On the other hand, it is known (see Introduction) that the cyclotron instability can develop in a pulsed mode even without external modulation. The simplest model of such a pulsed generation provides damped relaxation oscillations of the instability, which can most probably be observed if the system is disturbed by a single impulsive injection of energetic particles (e.g. during the substorm expansion phase). The theory of the relaxation oscillations (Bespalov and Trakhtengerts, 1976; Davidson, 1979) shows that they can have a rather high quality factor, i.e. be damped rather weakly.

In our case the precipitation is observed by EISCAT rather far from the injection region, where the injected cloud has already spread in longitude. Thus, the drift of this cloud through the instability region serves not as an impulsive source but as a quasi-stationary source with slowly varying parameters. The time scale of this variation is about the duration of the entire event. As was noted in the Introduction, even a steady energetic electron source can lead to a pulsating regime of the instability.

If the source parameters vary slowly compared with the pulsation period, then the pulsation properties vary correspondingly. Let us compare the anticipated variation with the observed one. Note that a preliminary comparison was performed by Demekhov et al. (1998) on the basis of a selfconsistent model of the cyclotron instability, generalised to the case of the source with varying characteristics, such as the drifting particle flux, particle energy, and pitch-angle anisotropy of the distribution function. It turned out that for the parameters corresponding to the conditions of our experiment (i.e. the radial distance, duration of injection, energy of drifting electrons, and the anisotropy variation typical for drift in the geomagnetic field) the model reproduces well the basic characteristics of observed pulsating precipitations. In particular, it gives the correct results for the observed average 
repetition period of the precipitation and its variation. Here, we can add more detail to this comparison. As it was noted in Sect. 4, in the pulses observed in the beginning of the event, the energy of precipitated particles increased during the pulse. Such a mode is characteristic for a source with high anisotropy of particles. These properties are quite consistent with the properties of electrons near the front of a drifting particle cloud. At the end of the event, on the contrary, the energy of precipitated particles decreased during the pulse development. Such a mode is characteristic for a source of energetic particles with a moderate anisotropy. Again, this is consistent with the expected properties of electrons in the middle of the drifting particle cloud. Note that this result is in accord with the conclusions by Åsnes et al. (2005) about the existence of an upper anisotropy limit. This limit determines the dependence between the maximum possible values of the flux and anisotropy in a drifting cloud: the higher the anisotropy, the lower the observed flux.

\section{Conclusions}

In this paper, we analyzed the local time dependence of the characteristics of an energetic electron cloud, injected during a weak isolated substorm in the vicinity of the geostationary orbit. It was shown that within the local time sector from 03:00 until, at least 09:00 MLT the longitudinal drift of the substorm energetic electrons with $90^{\circ}$ pitch-angles is described well by a simple model of drift in the dipole magnetic field. The anisotropy increase in the drifting particle cloud gives rise to the cyclotron instability and related pitchangle diffusion, which explains why the fluxes at perpendicular and small pitch-angles vary synchronously. The pitchangle diffusion causes precipitation of the particles, which in our case, occurs in the form of short pulses with the repetition period of about $100 \mathrm{~s}$. The basic properties of these pulsating precipitations can be explained on the basis of the selfconsistent model of the cyclotron instability, considering the source of the energetic particles with smoothly varying characteristics, corresponding to the properties of the cloud of drifting particles (Demekhov et al., 1998). Near the injection region, within the longitudinal sector of 01:00-03:00 MLT, the longitudinal drift velocity differs from that in the dipole magnetic field. For the adequate description of the particle motion in this sector, it is necessary to use a more complex model, taking into account, in particular, the non-stationary substorm electric field.

Acknowledgements. The authors are thankful to R. D. Belian for providing the data of energetic particle measurements on LANL spacecraft. EISCAT is an International Association supported by Finland (SA), France (CNRS), the Federal Republic of Germany (MPG), Japan (NIPR), Norway (NFR), Sweden (NFR), and the United Kingdom (PPARC). The work was supported by the Division of Physical Sciences of RAS (program "Plasma processes in the solar system"), RFBR grant \# 04-05-64955, and by INTAS grant \# 03-51-4132.
Topical Editor I. A. Daglis thanks two referees for their help in evaluating this paper.

\section{References}

Andronov, A. A. and Trakhtengerts, V. Y.: Kinetic instability of the external Earth's radiation belt, Geomagnetism and Aeronomy (in Russian), 4(2), 233-242, 1964.

Åsnes, A., Friedel, R. W., Stadsnes, J., Thomsen, M., Østgaard, N., and Cayton, T.: Statistical pitch angle properties of substorminjected electron clouds and their relation to dawnside energetic electron precipitation, J. Geophys. Res., 110, A05207, doi:10.1029/2004JA010838, 2005.

Belian, R. D., Gisler, G. R., Cayton, T., and Christensen, R.: High$\mathrm{Z}$ energetic particles at geosynchronous orbit during the great solar proton event series of October 1989, J. Geophys. Res., 97, 16 897-16 906, 1992.

Bespalov, P. A. and Trakhtengerts, V. Y.: Dynamics of the cyclotron instability in a mirror system, Fiz. Plazmy, 2(3), 396-406, 1976. [Sov. J. Plasma Phys., 2(3), 215-221, 1976].

Bespalov, P. A.: Self-modulation of radiation of a plasma cyclotron “maser", Pis'ma Zh. Eksp. Teor. Fiz., 33, 192-195, 1981. [JETP Lett., 33(4), 182-185, 1981].

Birn, J., Thomsen, M. F., Borovsky, J. E., Reeves, G. D., McComas, D. J., and Belian, R. D.: Characteristic plasma properties during dispersionless substorm injections at geosynchronous orbit, J. Geophys. Res., 102, 2309-2324, 1997.

Birn, J., Thomsen, M. F., Borovsky, J. E., Reeves, G. D., McComas, D. J., Belian, R. D., and Hesse, M.: Substorm electron injections: Geosynchronous observations and test particle simulations, J. Geophys. Res., 103, 9235-9248, 1998.

Cayton, T. E., Belian, R. D., Gary, S. P., and Fritz, T. A.: Energetic electron components at geosynchronous orbit, Geophys. Res. Lett., 16(2), 147-150, 1989.

Collier, M. R.: Are magnetospheric suprathermal particle distributions (kappa functions) inconsistent with maximum entropy considerations?, Advances in Space Research, 33, Is.11, 2108-2112, 2004.

Coroniti, F. V. and Kennel, C. F.: Electron precipitation pulsations, J. Geophys. Res., 75, 1279-1289, 1970.

Davidson, G. T.: Self-modulated VLF wave electron interactions in the magnetosphere: a cause of auroral pulsations, J. Geophys. Res., 84, 6517-6523, 1979.

Davidson, G. T.: Pitch angle diffusion in morningside aurorae, 2. The formation of repetitive auroral pulsations, J. Geophys. Res., 91, 4429-4436, 1986.

Davidson, G. T.: Pith angle diffusion and the origin of temporal and spatial structures inside morning aurorae, Space Science Reviews, 53, 45-82, 1990.

Demekhov, A. G., Lyubchich, A. A., Trakhtengerts, V. Y., Titova, E. E., Manninen, J., and Turunen, T.: Modeling of nonstationary electron precipitation by the whistler cyclotron instability, Ann. Geophys., 16, 1455-1460, 1998, http://www.ann-geophys.net/16/1455/1998/.

Friedel, R. H., Korth, A., and Kremser, G.: Substorm onsets observed by CRRES: Determination of energetic particle source regions, J. Geophys. Res., 101, 13 137-13 154, 1996.

Hamlin, D. A., Karplus, R., Vik, R. C., and Watson, K. M.: Mirror and azimuthal drift frequencies for geomagnetically trapped 
particles, J. Geophys. Res., 66, 1-5, 1961.

Heacock, R. R. and Hunsucker, R. D.: A study of concurrent magnetic field and particle precipitation pulsations, 0.005 to $0.5 \mathrm{~Hz}$, recorder near College, Alaska, J. Atmos. Terr. Phys., 39, 487501, 1977.

Higbie, P. R., Belian, R. D., and Baker, D. N.: High-resolution energetic particle measurements at $6.6 \mathrm{R}_{E}, 1$. Electron micropulsations, J. Geophys. Res., 83, 4851-4855, 1978.

Isenberg, P. A., Koons, H., and Fennel, J.: Simultaneous observations of energetic electrons and dawnside chorus in geosynchronous orbit, J. Geophys. Res., 87, 1495-1503, 1982.

Johnson, M. N. and Kierein, J.: The Combined Release and Radiation Effects Satellite (CRRES): Spacecraft and mission, Journal of Spacecraft and Rocets, 29, 556-563, 1992.

Johnstone, A. D.: The mechanism of pulsating aurora, Ann. Geophys., 1, 397-410, 1983,

http://www.ann-geophys.net/1/397/1983/.

Kennel, C. F. and Petschek, H. E.: Limit of stably trapped particle fluxes, J. Geophys. Res., 71, 1-28, 1966.

Korth, A., Kremser, G., Wilken, B., Guettler, W., Ullaland, S. L., and Koga, R.: The Electron and Proton Wide-Angle Spectrometer (EPAS) on the CRRES spacecraft, Journal of Spacecraft and Rocets, 29, 609-614, 1992.

Li, X., Sarris, T. E., Baker, D. N., Peterson, W. K., and Singer, H. J.: Simulation of energetic particle injections associated with a substorm on August 27, 2001, Geophys. Res. Lett., 30(1), CiteID 1004, doi:10.1029/2002GL015967, 2003.

Lopez, R. E., Sibeck, D. G., McEntire, R. W., and Krimigis, S. M.: The energetic ion substorm injection boundary, J. Geophys. Res., 95, 109-117, 1990.

Lyons, L. R. and Williams, D. J.: Quantitative aspects of magnetospheric physics, D. Reidel Pub. Co., 1984.

Manninen, J., Turunen, T., Lubchich, A., Titova, E., and Yahnina, T.: Relations of VLF emissions to impulsive electron precipitation measured by EISCAT radar in the morning sector of auroral oval, J. Atmos. Terr. Phys., 58, 97-106, 1996.

Mead, G. D. and Fairfield, D. H.: A quantitative magnetospheric model derived from spacecraft magnetometer data, J. Geophys. Res., 80, 523-542, 1975.

Nygrén, T., Kaila, K. U., Huuskonen, A., and Turunen, T.: Determination of $E$ region effective recombination coefficient using impulsive precipitation events, Geophys. Res. Lett., 19, 445-448, 1992.

Omholt, A. and Berger, S.: The occurrence of auroral pulsations in the frequency range $0.01-0.1 \mathrm{c} / \mathrm{s}$ over Troms $\emptyset$, Planet. Space Sci., 15(6), 1075-1080, 1967.

Østgaard, N., Stadsnes, J., Bjordal, J., Vondrak, R. R., Cummer, S. A., Chenette, D. L., Parks, G. K., Brittnacher, M. J., and McKenzie, D. L.: Global-scale electron precipitation features seen in UV and X rays during substorms, J. Geophys. Res., 104, 10 191$10204,1999$.

Østgaard, N., Stadsnes, J., Bjordal, J., Vondrak, R. R., Cummer, S. A., Chenette, D. L., Schulz, M., and Pronko, J. G.: Cause of the localized maximum of X-ray emission in the morning sector: A comparison with electron measurements, J. Geophys. Res., 105, 20 869-20 884, 2000.

Reeves, G. D., Henderson, M. G., McLachlan, P. S., and Belian, R. D.: Radial propagation of substorm injections, Third International Conference on Substorms (ICS-3), 579-584, 1996.
Roederer, J. G.: Dynamics of geomagnetically trapped radiation, Springer-Verlag, 1970.

Roederer, J. G. and Hones Jr., E. W.: Motion of magnetospheric particle clouds in a time-dependent electric field model, J. Geophys. Res., 79, 1432-1438, 1974.

Sandahl, I., Eliasson, L., and Lundin, R.: Rocket observations of precipitating electrons over a pulsating aurora, Geophys. Res. Lett., 7, 309-312, 1980.

Sarris, T. and Li, X.: Evolution of the dispersionless injection boundary associated with substorms, Ann. Geophys., 23, 877884, 2005,

http://www.ann-geophys.net/23/877/2005/.

Sato, N.: Short-period magnetic pulsations associated with periodic VLF emissions (T 5.6 s), J. Geophys. Res., 89, 2781-2787, 1984.

Sergeev, V. A. and Yahnin, A. G.: The features of auroral bulge expansion, Planet. Space Sci., 27, 1429-1440, 1979.

Sergeev, V. A., Pellinen, R. J., Bösinger, T., Baumjohann, W., Stauning, P., and Lui, A. T. Y.: Spatial and temporal characteristics of impulsive structure of magnetospheric substorms, J. Geophys., 60, 186-198, 1986.

Shukhtina, M. A. and Sergeev, V. A.: Modelling the drift of the real magnetosphere energetic particles in the vicinity of a geosynchronous orbit, Geomagnetism and Aeronomy (in Russian), 31(5), 775-780, 1991.

Takahashi, S. and Iyemori, T.: Three-dimensional tracing of charged particle trajectories in a realistic magnetospheric model, J. Geophys. Res., 94, 5505-5509, 1989.

Torkar, K. M., Riedler, W., Kremser, G., Korth, A., Ullaland, S., Stadsnes, J., Block, L. P., Iversen, I. B., Tanskanen, P., and Kangas, J.: A study of the interaction of VLF waves with equatorial electrons and its relationship to auroral X-rays in the morning sector, Planet. Space Sci., 35, 1231-1253, 1987.

Trakhtengerts, V. Y., Tagirov, V. R., and Chernous, S. A.: Flow cyclotron maser and pulsed VLF emissions, Geomagnetism and Aeronomy (in Russian), 26, 99-106, 1986.

Tsyganenko, N. A.: A magnetospheric magnetic fields model with a warped tail current sheet, Planet. Space Sci., 37, 5-20, 1989.

Vedenov, A. A., Velikhov, E. P., and Sagdeev, R. Z.: Quasi-linear theory of plasma oscillations, Nucl. Fusion Suppl., 2 (part 2), 465-475, 491-494, 822, 834-835, 858-859, 1962.

Walker, R. J., Erickson, K. N., and Winckler, J. R.: Pitch angle dispersion of drifting energetic protons at synchronous orbit, J. Geophys. Res., 83, 1595-1600, 1978.

Yahnin, A. G., Sergeev, V. A., Pellinen, R. J., Baumjohann, W., Kaila, K. U., Ranta, H., Kangas, J., and Raspopov, O. M.: Substorm time sequence and microstructure on 11 November 1976, J. Geophys., 53, 182-197, 1983.

Yahnin, A. G., Bosinger, T., Kangas, J., and Belian, R. D.: Some implications on substorm dynamics inferred from correlations between multiple flux peaks of drifting proton clouds and ground observations, Ann. Geophys., 8, 327-336, 1990, http://www.ann-geophys.net/8/327/1990/.

Yahnin, A., Titova, E., Lubchich, A., Bösinger, T., Manninen, J., Turunen, T., Hansen, T., Troshichev, O., and Kotikov, A.: Dayside high latitude magnetic impulsive events: their characteristics and relationship to sudden impulses, J. Atmos. Terr. Phys., 57, 1569-1582, 1995. 